\title{
Lattice Structure Design and Optimization With Additive Manufacturing Constraints
}

\author{
Yunlong Tang, Guoying Dong, Qinxue Zhou, and Yaoyao Fiona Zhao
}

\begin{abstract}
Lattice structures with different desired physical properties are promising for a broad spectrum of applications. The availability of additive manufacturing (AM) technology has relaxed the fabricating limitation of lattice structures. However, manufacturing constraints still exist for AM-fabricated lattice structures, which have a significant influence on the printing quality and mechanical properties of lattice struts. In this paper, a design and optimization strategy is proposed for lattice structures with the consideration of manufacturability to ensure desired printing quality. The concept of manufacturable element is used to link the design and manufacturing process. A metamodel is constructed by experiments and the artificial neural network to obtain the manufacturing constraints. Sizes of struts are optimized by a bidirectional evolutionary structural optimizationbased algorithm with these manufacturing constraints. An arm of quadcopter is redesigned and optimized to validate the proposed method. Its result shows that optimized heterogeneous lattice structures can improve the stiffness of the model compared to the homogeneous lattice structure and the original design. Both the Von-Mises stress and the maximum displacement are reduced without increasing the weight of designed part. And by considering the manufacturability constraints, the optimized design has been successfully fabricated by the selected additive manufacturing process.
\end{abstract}

Note to Practitioners-Lattice structures might fail to be fabricated by the additive manufacturing technique if the designed model exceeds the processability of the machine. Our approach has the capability of considering the manufacturing constraints in the design and optimization process. We conducted experiments to investigate the manufacturability and proposed a method that can give the domain of the design variables for a selected manufacturing process. And we also designed an algorithm that can optimize the lattice structure inside the domain of design variables. It ensures that the lattice model can be successfully fabricated by the selected process and the performance is dramatically increased compared to the original design. Engineers can use our approach to optimize the lattice structure automatically without knowing the knowledge of optimization and manufacturability.

Index Terms - Additive manufacturing (AM), design, lattice structure, manufacturing constraints.

Manuscript received October 4, 2016; revised December 8, 2016; accepted January 23, 2017. This paper was recommended for publication by Associate Editor C. C. L. Wang and Editor Y. Chen upon evaluation of the reviewers' comments. This work was supported in part by the National Sciences and Engineering Research Council of Canada Discovery under Grant RGPIN 436055-2013, in part by the China Scholarship Council under Grant 201306020032, and in part by the Rio Tinto-Richard Evans Fellowship in Engineering. (Corresponding author: Yaoyao Fiona Zhao.)

The authors are with the Mechanical Engineering Department, McGill University, Montreal, QC H3A 0G4, Canada (e-mail: yaoyao.zhao@mcgill.ca).

Color versions of one or more of the figures in this paper are available online at http://ieeexplore.ieee.org.

Digital Object Identifier 10.1109/TASE.2017.2685643

\section{INTRODUCTION}

$\mathbf{T}$ HE word "lattice" originally describes a framework or structure of crossed woods or metal strips and can be extended to regular geometrical arrangements of points or objects over an area and space [1]. In this paper, the lattice structure is defined as a mesoscale $(0.1-10 \mathrm{~mm})$ or microscale $(<0.1 \mathrm{~mm})$ truss-like structure which consists of interconnected struts and nodes with a certain repeated arrangement in 3-D space. In this sense, lattice structures can be regarded as a type of cellular structure on a mesoscale. Compared to other types of cellular structures including foams and honeycombs, lattice structures are more flexible to achieve a wide range of different desired physical properties, such as high stiffness-weight ratio [2], low thermal expansion coefficient [3], negative Poisson ratio [4], and high heat dissipation rate through active cooling [5]. Moreover, it can also be designed as a bioimplant to enhance the ossesointegration as well as alleviating stress-shielding effect. Due to its outstanding performance, lattice structures have been used in a broad spectrum of applications, including bone and dental implants [6]-[8], ultralight structures [9], [10], energy absorbers [11], low thermal expansion structures [12], and conformal cooling [13].

Traditionally, lattice structures can be fabricated via casting, sheet metal forming, or wire bonding processes [14]. However, manufacturing constraints of these processes severely restrict the complexity of designed lattice structures. These processes can only be applied to fabricate lattice structures with few simple unit cell topologies in a regular shape on a macroscale. This manufacturing limitation has been relaxed by using additive manufacturing (AM) to fabricate lattice structures. By fabricating a part layer by layer, AM enables the design of complex structures without significantly increasing the cost. Thus, the geometrical freedom provided by AM greatly enlarges the design space of lattice structures. Lattice structures with multiscale complexities can be easily fabricated for a better functional performance. For instance, on a macroscale, conformal lattice structures [15] can be produced to fit a complex macroshape with a relatively smooth surface boundary. On a mesoscale or microscale, complex lattice unit cells have been designed and fabricated to achieve a given gradient of elastic properties [16]. However, it should also be noted that every manufacturing process has limitations. AM is no exception. In particular, some recent research has observed and studied on the manufacturing constraints of lattice structures fabricated by different AM processes. The manufacturability of gyroid lattice structures made of $316 \mathrm{~L}$ stainless steel 


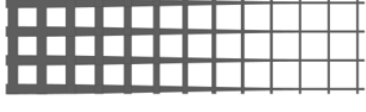

a

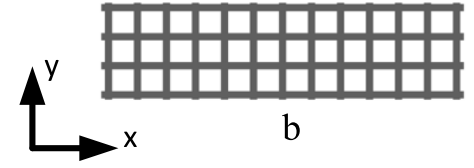

Fig. 1. Comparison between: (a) homogeneous lattice and (b) heterogeneous lattice [9].

with selective laser melting (SLM) process has been studied in [17]. In this research, the lattice structures with unit cell sizes ranging from 2 to $8 \mathrm{~mm}$ with $15 \%$ volume fraction can be successfully manufactured. Based on the fabrication experiments, it shows that the minimum unit cell size which can be fabricated mainly depends on particle sizes of metal powders, since the trapped powders with a big particle size in a small unit cell are difficult to be removed. Moreover, it also concludes that the unit cell whose size is larger than $8 \mathrm{~mm}$ is problematic to be fabricated due to the longer distance of the overhang area. Similar research has been done by Santorinaios et al. [18] on the lattice cells with vertical and cross struts fabricated by SLM process. This research also shows that the lattice structures with large cell size (larger than $5 \mathrm{~mm}$ ) are difficult to be fabricated. Since their struts tend to "slag" during the manufacturing process. To further evaluate the manufacturability of lattice structures with different geometrical configurations, a manufacturability table is used in [19]. In this research, two different topologies of lattice cells, gyroid and diamond, are fabricated by SLM process with Ti6Al4V powders. The building conditions of each type of cells with different cell sizes and volumes are recorded. The result shows the lattice structures with a low volume fraction and 4-5 mm cell sizes mostly failed. Instead of the study on some lattice topologies, design rules are provided by Wang et al. [20] to achieve a better manufacturability of $316 \mathrm{~L}$ lattice structures fabricated by SLM process. Several set of benchmark parts have been designed to individually evaluate the effects of inclined angles and sizes of minimum struts on the manufacturability of lattice structures [20]. Based on these benchmarks, a design guideline shows that the struts with inclined angles smaller than $35^{\circ}$ are hard to fabricate. It also indicates that the minimum fabricatable size of struts is $0.15 \mathrm{~mm}$.

To help designers consider both freedom and constraints of AM processes for lattice structures, several design methods for lattice structures have been proposed. Generally, these design methods can be divided into two groups: the design of homogeneous lattice structures and the design of heterogeneous lattice structures. As it is shown in Fig. 1(b), a homogeneous lattice structure consists of unit cells with exactly the same shape periodically distributed in the design space. Thus, this type of lattice structures can be considered as homogeneous materials on a macroscale during the design process. Compared to the shapes and sizes of cells in homogeneous lattice structures, those of cells in heterogeneous lattice structures may vary from point to point to achieve a certain type of functional gradient. A typical example of heterogeneous lattice structures is shown in Fig. 1(a). By varying thickness of struts, this lattice structure can achieve a certain elastic properties gradient along the $x$-axis.

For homogeneous lattice structure, most of research is focusing on its unit cell. Topology optimization and homogenization techniques can be applied to generate the lattice unit cell which can achieve the desired properties. In order to achieve the fabricatable unit cell, the manufacturing constraints such as the minimum member size [21] can be considered in the optimization procedure of microcell structure. To further improve the functional performance of homogeneous lattice structures, some concurrent optimization methods [22]-[24] have been developed to update the cell topology as well as the macroshape of lattice simultaneously. Compared to other methods only focusing on single design scale, these concurrent methods generally show a better performance on multifunctional applications [22].

Compared to homogeneous lattice structures, heterogeneous lattice structures generally provide more design freedom. Designers can control the distribution of some lattice parameters to achieve a better functional performance. To achieve this goal, several design and optimization methods for this type of lattice structures are proposed. A general framework for lattice structures fabricated by AM processes is proposed in [10]. This general framework can be divided into two stages. In the first stage, size optimization is applied to optimize the size of lattice struts for a better mechanical performance. In the optimization procedure, the size of lattice struts is constrained in a predefined range based on the manufacturability of the machine. Then in the second stage, another optimization process is applied to obtain the optimal process parameters for each strut based on the proposed concept manufacturable element (ME). Besides the size optimization, several density-based topology optimization methods [25]-[30] have also been applied to design the heterogeneous lattice or cellular structures for a better mechanical performance. Among them, different types of material interpolation functions are used to establish the relationship between the properties and parameters of mesoscale structures. Based on these interpolation functions, a topology optimization problem is formalized and solved to get the optimal relative density distribution. Then, the mapping functions are further used to convert the result of topology optimization to the distribution of the lattice relative density. In the mapping functions of some proposed methods, like Rosen's method, predefined manufacturing constraints are considered. In alternative of density-based optimization methods, bidirectional evolutionary structural optimization (BESO) method [9] has also been modified and applied to design heterogeneous lattice structures. This method tries to simulate the bone remolding process by moving materials from low stress struts to high stress struts. Compared to density-based optimization methods, BESO-based method can easily deal with those stress-related constraints during the optimization process. To further reduce the size of design variables, a heuristic optimization method has been developed by Nguyen et al. [31]. This method generally converts the original problem into the optimization process with two design variables: maximum and minimum sizes of struts. Then, size matching and scaling method have been applied to determine the size of each strut in the design domain 
with the consideration of local stress states. Like other methods mentioned here, predefined upper and lower bounds of struts' size are considered as constraints from manufacturing.

In summary of those existing design and optimization methods of lattice structures for AM, it is notable that the functional performance of parts can be further improved by using heterogeneous lattice structures. Moreover, as to the manufacturability of AM-fabricated lattice structures, most design methods only simply consider the constraints on the thickness of struts. However, other geometrical parameters such as the inclined angle [20] and the horizontal overhang [17] may also affect the fabrication quality and manufacturability of lattice structures, and the effects of those parameters are not totally independent. Even though some design rules [20] have been provided, most of them failed to consider the interaction between different geometrical parameters. Thus, it is difficult to directly integrate those rules into a general design and optimization process of lattice structures.

To solve the mentioned issues, a design and optimization method of lattice structures is proposed in this paper with the consideration of manufacturability constraints of AM processes. In this method, a concept called ME is used to link the design and manufacturing process. To predict the quality and manufacturability of each ME, the artificial neural network (ANN) is established as a meta-model for the selected AM process for a certain type of material. Based on the predicted value, the manufacturing constraint of each ME can be obtained. Then, obtained constraints will be used in the BESO-based lattice optimization process to get the optimal size distribution of lattice struts. In this paper, fused deposition modeling (FDM) process, one of the most widely used AM processes for thermal plastic polymers, is used as an example to illustrate the proposed method. However, it should be noted that this methodology can be further applied to other different types of AM processes with different materials. Moreover, compared to existing design methods, the proposed method can automatically consider the effects of different geometrical parameters on the manufacturability of lattice structures in the optimization procedure. Thus, it can guarantee the processablity of designed lattice structures.

In order to clearly describe the proposed method, this paper is organized as follows. In Section II, several basic concepts which are used in the proposed method are presented first. Based on these concepts, the proposed design method and its related meta-model of the manufacturing process are carefully explained in Section III. In Section IV, a case study is used to further illustrate and validate the proposed method, and a discussion on the design results under different types of constraints is provided. At the end, this paper is wrapped up with conclusions and future research directions.

\section{BASIC CONCEPTS}

\section{A. Physical Entity}

To represent the design space of lattice structures, the concept named as physical entity is used in this paper. According to the previous research [32], physical entity is defined as a concrete entity which is implemented for physical behaviors required by given functions. Physical entity mainly consists

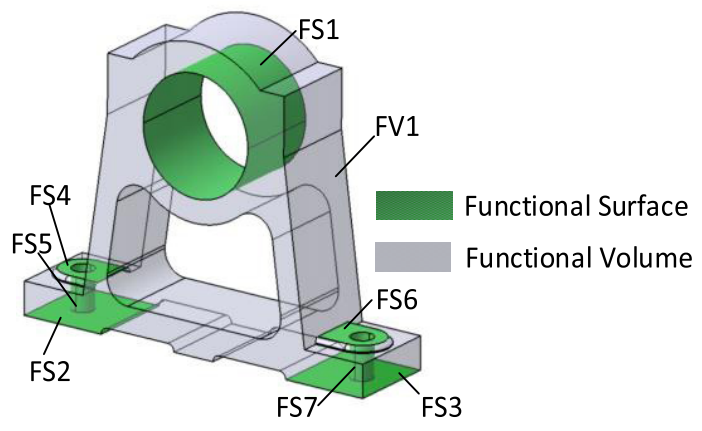

Fig. 2. Example of FSs and FVs [35].

of the information of the design boundary for the detailed design stage. It can be considered as the input for the proposed design method. This input can be obtained from the functional design step in the conceptual stage [33] or directly extracted from the existing parts. Generally, a physical entity should include two types of information: geometrical information and material information. Material information includes a set of feasible materials which can achieve the defined functions of a physical entity. In most cases, the shape of physical entity is only defined on a macroscale. As to the information related to mesostructure or microstructure of physical entity, they are also considered as the material information in this paper.

Besides the material information, concepts of functional surfaces (FSs) and functional volumes (FVs) [34] are used to represent the geometrical information of a physical entity. In this paper, an FS is referred to the key surface of a physical entity to realize the requested functions, such as assembly surfaces or loading surfaces. An FV is a geometrical volume of a physical entity which is used to link the surfaces and assist FSs to achieve the required functions. A typical example of FSs and FVs is provided and shown in Fig. 2. In this figure, those green-tagged surfaces represent FSs, while the FV is shown in the gray color to connect those FSs. The FSs of this example are designed for three different purposes. The bottom two surfaces are used to provide a vertical support. Another two cylindrical surfaces with connected surfaces on their top are designed for the assembly of bolts. The cylindrical hole on the top of the part is used to hold a bearing. It should be noted that in some design cases, the FSs or FVs for a given physical entity after the conceptual design stage is not totally fixed. For these design cases, the parametric representation for FSs and FVs is needed. The detailed information of parametric representation is beyond the scope of this paper. A detailed discussion of parametric representation of FV can be found in [33].

\section{B. Lattice Unit Cell Model}

To represent the topology of a repeating element inside the lattice structures, lattice unit cell model is proposed. In this model, the node of a lattice cell is defined in a unit cubic space shown in Fig. 3, which can be represented by a 3-D tuple $p$

$$
p=\left(e_{1}, e_{2}, e_{3}\right) \in P \subseteq[0,1]^{3}
$$

where $e_{i}$ is the $i$ th component of the tuple and $P$ is the set which contains all the nodes of a lattice unit cell. To represent 


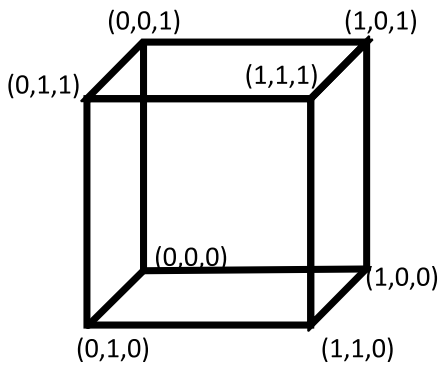

Fig. 3. Unit cubic defined in a parametric space.

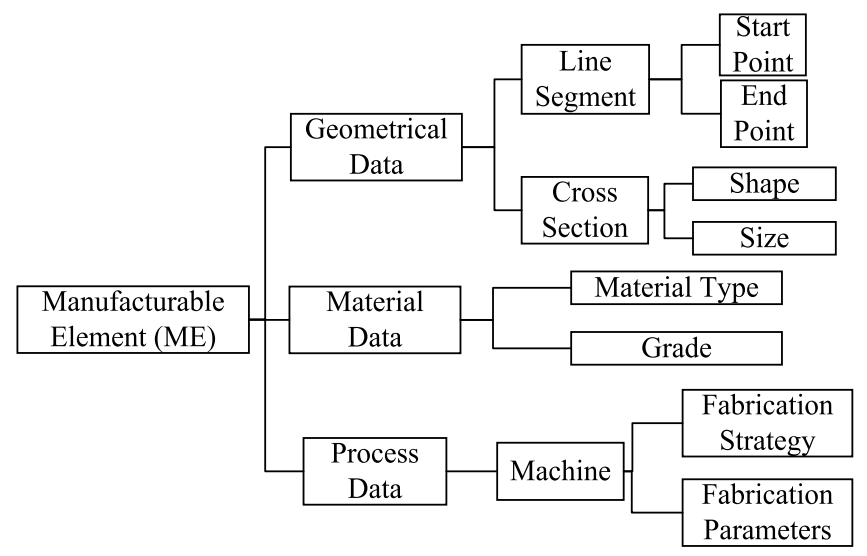

Fig. 4. Graphical view of data structure of ME for lattice structures.

each strut of lattice unit cell, the node pair $l$ is used and expressed as

$$
l=\left\{p_{i}, p_{j}\right\}, \quad p_{i} p_{j} \in P .
$$

Based on the definition of lattice nodes and struts, the topology of lattice unit cell can be expressed as a unidirectional graph $G$

$$
G=\{P, S\}
$$

where $S$ is the set that contains all the struts inside the lattice unit cell. According to different design requirements, different lattice unit cell topologies are selected by designers. Based on the defined lattice unit cell model, lattice frame which represents the topological skeleton of lattice structures can be built. This process is discussed in Section III-C.

\section{Manufacturable Element of Lattice Structures}

To link the design and manufacturing process, a concept called ME is used in this paper. This concept is originally defined in [10] as a predefined, parametrized decomposition of a volumetric region of a part. Based on this original concept and the characteristics of lattice structures focused in this paper, an ME of lattice structure is defined as a lattice strut with its related geometry, material, and process information. To parametrically represent each ME of lattice structures in the proposed design method, a data structure of $\mathrm{ME}$ is proposed and its graphical view is shown in Fig. 4.

It is clear that $\mathrm{ME}$ of lattice structures consists of three types of data. They are geometrical data, material data, and process data. The geometrical data of ME can be further divided into two categories: the line segment and the crosssectional shape. A line segment consists of a pair of nodes

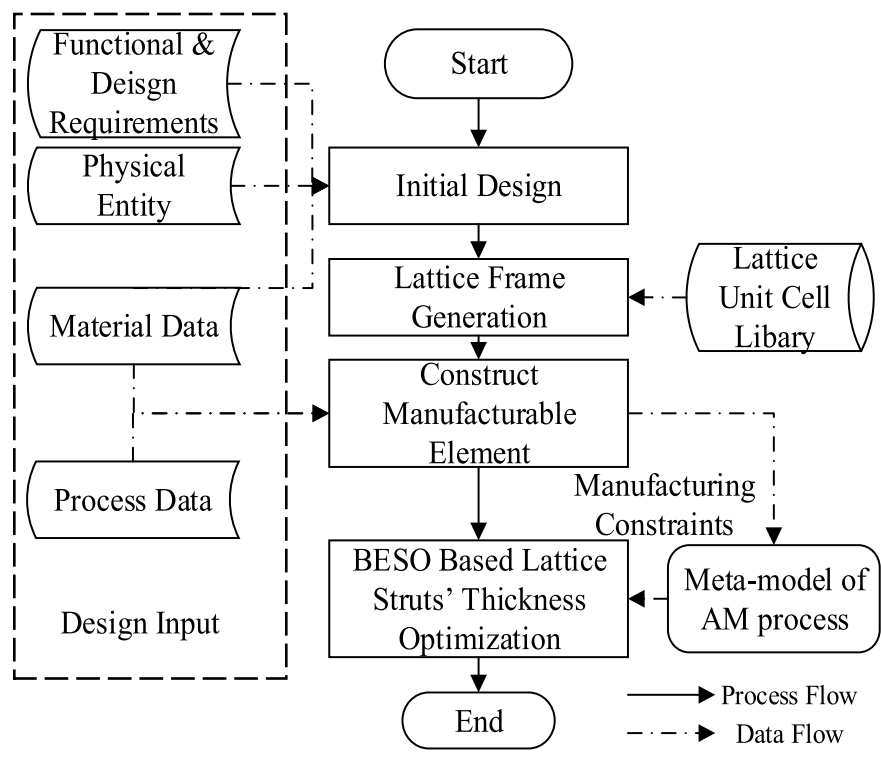

Fig. 5. General design flow of proposed design method.

to represent the midline of a strut, while the cross section can be defined by the shape and its related parameters, such as the diameter of a circle or the size of a square. Besides geometrical data, the material data are another very important factor. To clearly describe material compositions of fabricated lattice struts, both the material type and its grade should be included in the data model of ME. The last key factor for ME is the process data. It should be noted that different AM machines may have different manufacturing capabilities, even though they belong to the same type of AM technologies. Thus, as to process data of ME, a certain AM machine should be determined first. Then, based on the selected machine, its related fabrication strategy and fabrication parameters can be decided. Among these three types of data, the geometrical data are the primary concern of the proposed design method in this paper. Thus, they have been considered as design variables during the optimization process. As to material data and process data, they can both be decided during the early conceptual design stage based on material selection and process selection strategy. Thus, these two types of information are regarded as the input of the proposed design method.

\section{Methodology}

\section{A. General Design Flow}

The general design flow of the proposed design method is shown in Fig. 5. As it is shown in this figure, four types of data are considered as the input of the proposed design method. Among them, functional and design requirements are the most important factors, since it should be determined at the beginning of the whole design process. This type of data describes the functional behaviors and the related requirements of a designed part or product. Based on the functional and design requirements, other three types of data can be obtained from the conceptual stage. The general goal of this proposed method is to generate a manufacturable lattice structure with a better performance under given functional and design requirements. To achieve this purpose, the general 
design flow of the proposed design method is given and shown in Fig. 5. Its entire process can be generally divided into four steps which are discussed in the following sections, respectively. Besides these four major steps, an ANN-based meta-model of selected AM process is built and applied to obtain the manufacturing constraints for each ME during the design and optimization process. Thus, this model and its related experiments are also going to be explained in detail.

\section{B. Initial Design}

In the initial design step, some macroscale design parameters such as the weight or volume target for each FV should be determined. These parameters will be regarded as the design constraints in the following optimization step. To achieve this purpose, the density-based topology optimization is used in the initial design stage. Specifically, for a design problem of a minimum compliance with a constrained volume, the mathematical formulation of density-based topology optimization can be expressed as

$$
\begin{aligned}
& \text { Find: } \mathbf{x} \in R^{n}, \mathbf{x}=\left(x_{1}, x_{2}, \ldots, x_{n}\right) \\
& \text { Minimize: } c=f^{T} u \\
& \text { s.t.: } \quad K(x) u=f \\
& \quad \sum x_{i} v_{i} \leq V \\
& \quad 0<\varepsilon<x_{i} \leq 1
\end{aligned}
$$

where $\mathbf{x}$ represents an $n$-D vector which contains the relative density of each element inside the FVs of physical entity; $c$ is the structural compliance which can be calculated from the global external force vector $f$ and a global displacement vector $u ; K(x)$ is the global stiffness matrix; $v_{i}$ is the volume for $i$ th element, and $V$ is the upper limit of the total volume; $\varepsilon$ is a small positive value which is slightly larger than 0 to avoid the singularity of the stiffness matrix during the optimization process. In this paper, $\varepsilon$ is selected as 0.001 . To solve the topology optimization problem defined in (1), several methods including solid isotropic material with penalization (SIMP) [36] can be applied. Based on these methods, the optimal relative density distribution can be obtained. This distribution can be used as a reference to determine the volume target for each FV. Particularly, for $\mathrm{FV}_{j}$, its volume target $V_{\mathrm{FV}_{j}}$ can be calculated based on the following equation:

$$
V_{\mathrm{FV}}=\sum x_{i} v_{i}
$$

where $x_{i}$ is the volume of the $i$ th element inside $F V_{j}$, and $v_{i}$ is its optimal relative density obtained from topology optimization. For those FVs with solid material, this volume constraint can be achieved by removing materials in the low relative density area like traditional topology optimization routine. As to those FVs filled with lattice structures, the calculated volume constraint from (5) can be used to calculate the struts' thickness of homogeneous lattice structures which will be regarded as the initial design in the BESO-based lattice optimization method. Since BESO-based optimization method used in this paper only moves materials from the low stress region to the high stress region, it can guarantee that the total volume of lattice structures unchanged during the

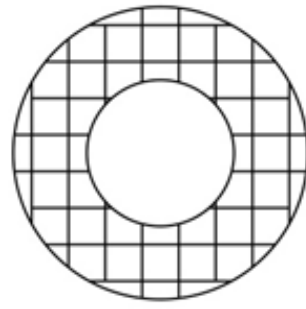

(a)

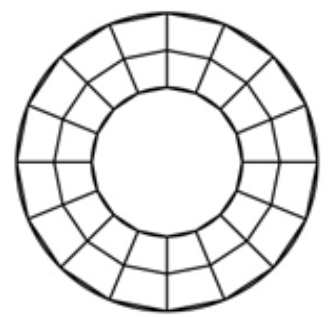

(b)
Fig. 6. Comparison between: (a) uniform lattice and (b) conformal lattice [9].

design optimization process. Generally speaking, the volume constraints generated in the initial design stage provide a foundation for the following optimization process.

\section{Lattice Frame Generation}

In the second step of the proposed design method, lattice frame which represents the topological skeleton of lattice structures is generated for the given FVs. To achieve this objective, several parameters including type of lattice frame and size of lattice cell need to be determined. Generally, lattice structures can be divided into two different types according to its frame configuration. They are uniform lattice structures (also known as periodic lattice structures) and conformal lattice structures. A comparison between these two types of lattice frames is given in Fig. 6. As it is shown in Fig. 6, the lattice frame of uniform lattice structures consists a periodically distributed lattice cell. Thus, each cell in the uniform lattice has the same size, shape, and topology. As to the conformal lattice, the size and shape of lattice cell's frame may vary to adapt to the macroshape of design boundary. Compared to uniform lattice structures, conformal lattice structures can guarantee the integrity of cells on the boundary of an FV. Thus, it can avoid some poorly connected struts located on the boundary of FV which are shown in Fig. 6(a). However, it should be noted that to generate conformal lattices for an FV with a complex geometry is not an easy task. Even though there is some research on conformal lattice generation [32], [37], most of them are limited to simple or regular shape. Moreover, the property of uniform lattices is comparatively easy to be controlled by carefully choosing the right cell size and topology. To consider both advantages and disadvantages of those two types of lattice structures, a general guideline is provided here to assist designers in decision-making. Generally, if an FV is bounded by the FSs which play roles as aesthetic or assembly purposes, then this FV is suggested to be filled with conformal lattice structures. Otherwise, uniform lattice structures can be used.

After the determination of lattice types, the topology of the lattice unit cell also needs to be determined. For both uniform and conformal lattice structures, the topology of a unit cell can be selected from the cell library which is a database for unit cell models. In this database, different lattice cell topologies are linked to different types of properties. For example, based on the loading condition of lattice struts, lattice cells can be divided into two types: bending dominant lattice and stretching dominant lattice [38]. In the structural design for a better 
stiffness, stretching dominant lattice cell is preferred, since the strut of this type of lattice is only under an axial load, which may exhibit better stiffness than its counterpart. However, in the design of compliant structures or energy absorption structures, bending dominant lattice structures are preferred. To further assist designers in selecting a suitable lattice unit cell from the unit cell library, material selection strategy [39] can also be used based on the material chart with effective properties of different cell topologies.

The size of lattice structure is another parameter needs to be considered in the current design stage. The size of the uniform lattice structure can be precisely controlled by three independent parameters. They are sizes on $X, Y, Z$-axes of the cell coordinate system. As to conformal lattice structures, their size is defined as a diameter of a circumscribed sphere of bounding box of lattice cell. Unlike uniform lattice structure, the size of conformal lattice structures cannot be accurately controlled, since both size or shape of lattice cells may vary inside the FV. Thus, in this design step, an average size of conformal lattice is used. To help designers select an appropriate cell size, two design guidelines are provided.

Guideline 1: The lattice cell size is suggested to be smaller than the minimum size of functional features.

Guideline 2: The lattice cell size $l$ is suggested to satisfy the following condition:

$$
p\left(l, t_{\min }\right)<\rho_{l}^{*}
$$

where the $\rho_{l}^{*}$ is the upper bound of the minimum relative density that lattice structures can achieve; $t_{\min }$ is a vector which contains the minimum fabricatable thickness of each strut in the lattice unit cell. Once the cell topology and process data are determined, the value of vector $t_{\min }$ is fixed. $p(l, t)$ is the function to calculate the relative density of a given lattice cell. The form of this function varies between cells with different topologies and struts' cross-sectional shape. However, they all depend on two independent variables: cell size $l$ and a vector $t$ which contains the thickness of each strut inside the cell. For example, the function $p_{\text {cubic }}(l, t)$ which is used to calculate the relative density of cubic cell with struts in square cross-sectional shape can be expressed as

$$
p_{\text {cubic }}(l, t)=1-3\left(1-\frac{t}{l}\right)^{2}+2\left(1-\frac{t}{l}\right)^{3}
$$

In (7), all the struts are assumed to have the same thickness. Thus, in this equation, $t$ is a scalar which represents the uniform thickness of struts in a lattice unit cell.

These two guidelines generally provide two limitations on the size of a lattice cell. Based on the first guideline, it can be concluded that the cell size cannot be too large. If it is larger than the minimum size of functional features, the lattice structures cannot be guaranteed to fully fill every corner of the design space. In the other word, there might be some void regions in the design space when the first guideline is violated. Designers will lose controllability on those regions in the subsequent optimization process. The second guideline provides a lower bound of cell size based on the manufacturability of selected AM process. Once the AM process and material are determined, the minimum fabricatable strut's thickness is fixed. The decreasing of the cell size will significantly increase the minimum achievable relative density. It limits the freedom of designers in the sebsequent optimization process. Moreover, it will also make the lattice structures lose the porosity which leads some issues on the simulations; therefore, the value of $\rho_{l}^{*}$ defined in (6) is suggested to be equal or smaller than 0.3 for efficient optimization as well as simulation. It should also be noted that the change of cell size inside the bounded region defined by the above two guidelines will lead slight change of the optimal functional performance. In order to achieve the optimal performance, designers can do the optimization with several different cell sizes and then select the cell size which can achieve the best performance.

Based on those design parameters determined at the beginning of this step, the lattice frame can be generated inside the design space. The general lattice frame generation method is given in this paper. Its process can be further divided into two substeps. In the first substep, hexahedron primitives can be generated inside the given FVs. For a uniform lattice structure, its hexahedron primitives should be exactly periodic. Thus, the kernel generation method discussed in the previous research [9] can be used. As to conformal lattice, the volume meshing technique can be used to generate hexahedron primitives in FVs with regular geometry. For an FV volume with complex geometry, auxiliary volume [32] can be built to convert a complex geometry into a simple and mapped shape. Since both ways have been exhaustively discussed in [9] and [32], this paper will not repeat them again.

In the second substep, the selected lattice cell topology can be populated into the generated primitives from the last substep. The generated hexahedron primitive can be represented by the positions of eight corners which are denoted as $C_{i j k}$, $i, j, k=0,1$. Once the positions of eight corners of hexahedron primitive are determined, the positions of all the lattice node inside this hexahedron primitive can be calculated by a tri-linear interpolation function. For example, the position $c_{p}$ of node $p$ inside the given hexahedron primitive $C_{i j k}$ can be calculated by

$$
\begin{aligned}
c_{j k} & =c_{1 j k} e_{1}+c_{0 j k}\left(1-e_{1}\right) \\
c_{k} & =c_{1 k} e_{2}+c_{0 k}\left(1-e_{2}\right) \\
c_{p} & =c_{1} e_{3}+c_{0}\left(1-e_{3}\right)
\end{aligned}
$$

where $\left(e_{1}, e_{2}, e_{3}\right)$ is the 3-D tuple represents the node $p$ inside the lattice unit cell model discussed in Section II. After calculating the position of each node, the frame of lattice struts can be built based on unidirectional graph $G$ by linking the related nodes. Finally, to trim those connected struts with the boundary of FV, the final frame of lattice structures can be obtained. To illustrate these two substeps of lattice frame generation, an example of arc shape FV is given and shown in Fig. 7.

\section{Manufacturable Element Construction}

To link the design and manufacturing process, a concept of ME is used in this paper. As mentioned in Section II-C, each ME mainly consists of three types of information. They are geometrical data, material data and process data. Both material 


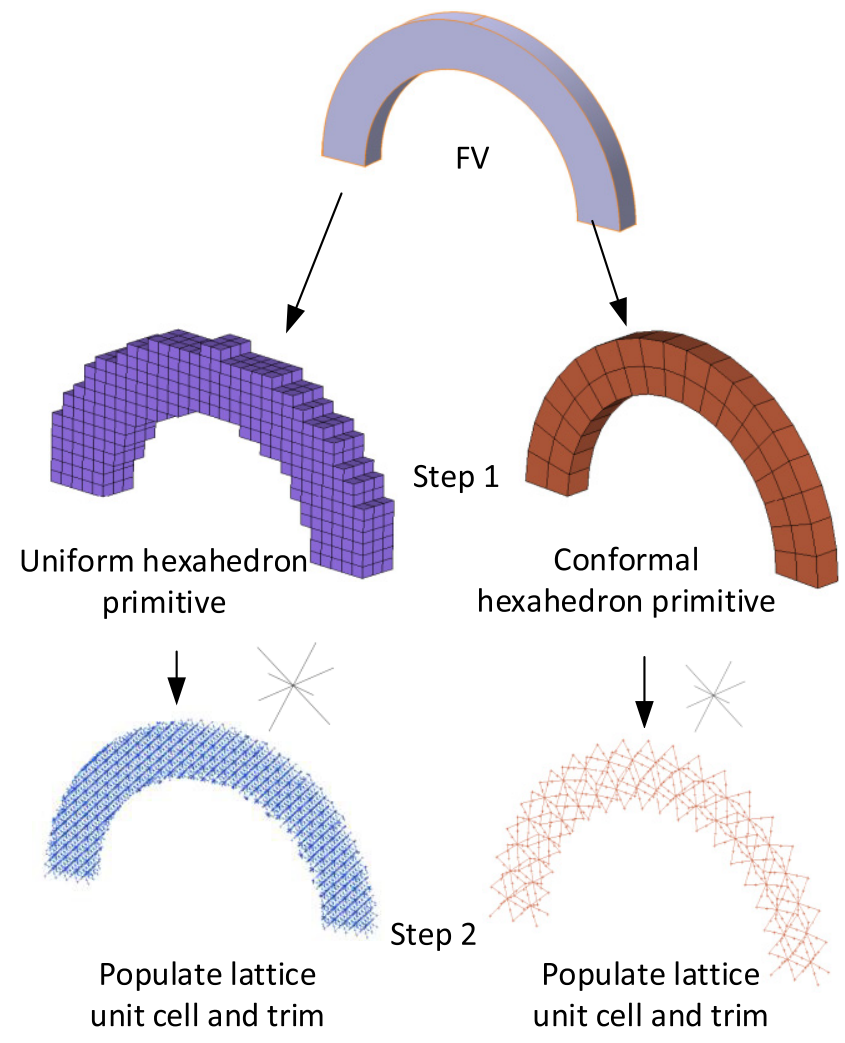

Fig. 7. Lattice frame generation.

and process data can be obtained as the input of the proposed design method. Thus, in this section, the focus is on how to build geometrical data based on the input lattice frame and physical entity.

As shown in Fig. 4, the geometrical data of lattice ME can be further divided into two types of data. They are line segments to represent the midline of the strut and its cross section. It should be noted that the line segment of the lattice ME is defined in the processing coordinate system rather than the design coordinate system where the lattice frame is generated. Thus, to construct the line segment of ME, the transformation matrix between the design coordinate system and the processing coordinate system is needed. This transformation matrix can be easily calculated once the fabrication orientation is determined. To simplify the process, the FVs with lattice structures will be considered as a solid volume temporarily. Based on this simplification and summarization of existing research related to orientation determination, two general guidelines are provided in this paper. These two general guidelines should be considered sequentially.

Guideline 1: To select the orientation which can guarantee the geometrical quality of FSs.

Guideline 2: To select the orientation with minimal volume of support structures.

Based on the processing orientation, a lattice strut can be easily converted to a line segment for the geometrical data of ME. As for cross-sectional data, the shape of the cross section can be predefined by users. But, it is impossible to finally decide the size of a lattice strut in current step. Thus, instead of using one value as the size parameter, a set of all the possible sizes of a lattice strut is set as the size parameter in this step. The manufacturing quality of this set of size parameters will be evaluated based on the proposed meta-model discussing in the following section. Based on the predicted results, a subset of them which can satisfy the quality requirement is chosen as the design space of BESO-based lattice optimization algorithm discussed in Section III-F.

\section{E. Meta-Model for AM Constraints}

The objective of using meta-model in this research is to obtain the manufacturing constraints for each ME during the design and optimization process. To simplify the process of getting meta-model, only the influence of the geometrical parameters on the manufacturability is considered in this paper. Therefore, the material and process parameters are set to be constant in the whole process. Based on the minimum recognition of the machine and the properties of lattice structure, like porosity, a design domain of geometrical data of MEs is obtained. This predefined domain is imported to the metamodel, and by setting printing quality in the meta-model, the design domain is adjusted to satisfy this printing quality. The meta-model is expressed as

$$
W=\Gamma(U, q) ; \quad W \subset U
$$

where $U$ is the predefined design domain, $q$ is the required manufacturing quality, $\Gamma$ represents the function of the metamodel, and $W$ is the design domain with the consideration of manufacturing constraints.

To obtain the meta-model of a selected AM process, several experiments are conducted to get the raw data of the printing quality at first. To obtain the relation between design parameters of lattice struts and its geometrical deviation, ANN model trained by the obtained raw data is utilized in this paper. For different types of MEs, the geometrical data could be different. Multiple ANNs would be trained by different categories of raw data. Then, the meta-model of a selected AM process is constructed by the trained ANNs. In this paper, FDM process is selected to fabricate samples for the experiment, and the obtained meta-model is only valid for this manufacturing process. However, the method of constructing the meta-model can be implemented on other AM process.

\section{1) Design of Experiment:}

a) Geometrical parameters of the lattice structure: In the experiment, three types of geometrical data, the diameter $D$, the length $L$, and the inclined angle $\theta$ are defined to build the ME. $D$ is the size of the cross section of the ME, $L$ is the length of the line segment of the ME, and $\theta$ is the angle between the line segment of the $\mathrm{ME}$ and the printing platform. The formulation of $L$ and $\theta$ are defined as

$$
\begin{aligned}
& L=\left|\overrightarrow{p_{s}}-\overrightarrow{p_{e}}\right| \\
& \theta=90^{\circ}-\left|\cos ^{-1} \frac{\left(\overrightarrow{p_{s}}-\overrightarrow{p_{e}}\right) \cdot \vec{z}}{\left|\overrightarrow{p_{s}}-\overrightarrow{p_{e}}\right|}\right|
\end{aligned}
$$

where $p_{s}$ and $p_{e}$ are the position of the start point and endpoint of the strut, $\vec{z}$ is the unit vector perpendicular to the printing platform.

According to the different tool paths shown in Fig. 8, lattice struts can be divided into three types, the horizontal 


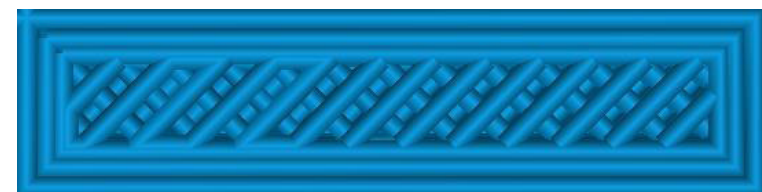

(a)

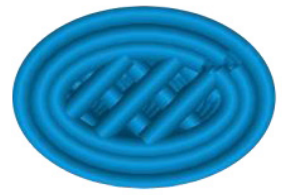

(b)

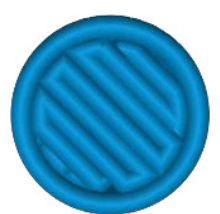

(c)
Fig. 8. Tool paths for different types of struts. (a) Horizontal strut (b) Inclined strut. (c) Vertical strut. (a)

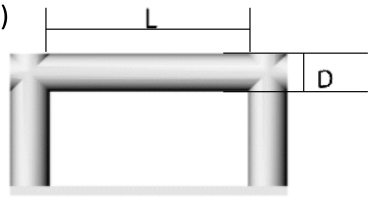

Horizontal Group

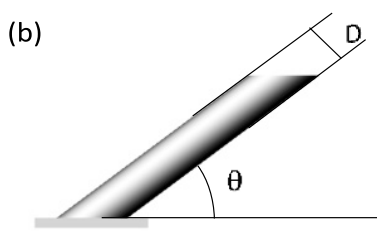

Non-horizontal group
Fig. 9. Models of test samples in the experimental and geometrical data. (a) Horizontal group. (b) Nonhorizontal group.

struts $\left(\theta=0^{\circ}\right)$, vertical struts $\left(\theta=90^{\circ}\right)$, and slanted struts $\left(0^{\circ}<\theta<90^{\circ}\right)$. Because the tool path of vertical struts and slanted struts are similar, vertical struts can be seen as a special case of slanted struts with $90^{\circ}$ inclined angle. Therefore, the experiment contains two groups of samples: one is the horizontal group, and the other is the nonhorizontal group, as shown in Fig. 9.

After doing the preliminary test of the selected FDM machine, proper values of defined geometrical data are chosen for the experiment to find the relationship between the manufacturability and these data. For the horizontal group, $L$ has six levels from 10 to $60 \mathrm{~mm}$. For the nonhorizontal group, $\theta$ has nine levels from $10^{\circ}$ to $90^{\circ}$. And for both groups, $D$ has five levels from 1 to $5 \mathrm{~mm}$. It should be noted the number of experimental groups is not always fixed. If the trained ANN model is not accurate enough, more groups of experiments can be added in those critical regions following the procedure as the initial set of experiments. These extra experiments can further refine the ANN model and make it more accurate especially on those highly nonlinear regions.

b) Manufacturing process: This paper concentrates on the influence of manufacturing constraints on topology optimization under a certain fabricating condition. Therefore, manufacturing parameters are constants during the whole printing process. FDM process is used to fabricate the MEs. The STereoLithography (STL) file is sliced by Z-Suite software. And the FDM printer is Zortrax M200. The printing material is Z-ABS [40]. All the process parameters are summarized in Table I. The printing speed of M200 only has two levels without a specific value, and normal level is selected in this paper. To get a more reliable result, each sample is printed three times, and the result is obtained from the average value.
TABLE I

PROCESS PARAMETERS

\begin{tabular}{|l|l|l|l|l|}
\hline Material & $\begin{array}{l}\text { Layer } \\
\text { Thickness }\end{array}$ & Fill Density & $\begin{array}{l}\text { Support } \\
\text { structure }\end{array}$ & $\begin{array}{l}\text { Printing } \\
\text { speed }\end{array}$ \\
\hline Z-ABS & $0.19 \mathrm{~mm}$ & Maximum & No & Normal \\
\hline
\end{tabular}

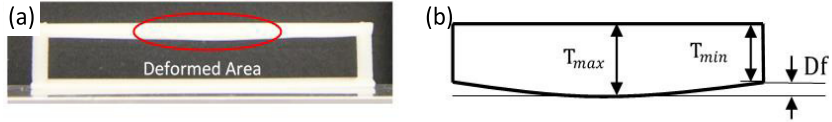

Fig. 10. Deflection of horizontal struts. (a) Deformed area. (b) Definition of $D f$.

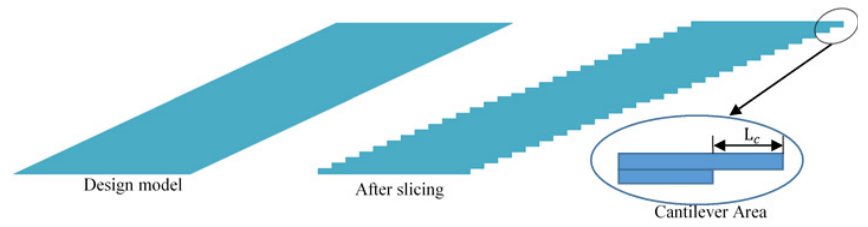

Fig. 11. Cantilever area of slanted struts.

\section{2) Measurement and Result:}

a) Criteria of manufacturability: To find the manufacturing constraints for lattice structures under a certain fabricating process, proper criteria of manufacturability should be predefined. There are several critical issues for horizontal struts and slanted struts. For horizontal struts, while printing the first layer of overhang struts, the filament is melt and extruded by the nozzle at a high temperature, so it cannot be cooled immediately. Because of no support structure, the first layer tends to deform due to its gravity and the deposition force of the second layer. Therefore, the thickness in the middle of the strut is larger than its two ends, which is shown in Fig. 10(a). If the deflection of the bottom is too large, the geometry will be inconsistent with the design model. Consequently, the deflection can be a criterion for horizontal struts. The deflection, which is shown in Fig. 10(b), is defined as

$$
D f=T_{\max }-T_{\min }
$$

where $D f$ is the deflection of the strut, and $T_{\max }$ and $T_{\min }$ are the maximum and minimum thickness of the strut.

For slanted samples, the difficulty is concentrated on the cantilever area of each new layer, which is shown in Fig. 11. With the inclined angle decreasing, the length of the cantilever area $L_{c}$ is getting longer. It is encountering the same problem as the first layer of the horizontal strut. $L_{c}$ is calculated by

$$
L_{c}=t / \sin \theta
$$

where $t$ is the thickness of each layer, $\theta$ is the inclined angle of the slanted strut.

$L_{c}$ will affect the thickness of the strut in certain directions. $t_{a}$ and $t_{b}$ are defined as the thickness of the strut in two directions to illustrate the different influence of $L_{c}$, as shown in Fig. 12. The thickness in certain direction is significantly influenced by $L_{c}$, as shown in Fig. 12(b). But in some directions, the phenomenon is not obvious, as shown in Fig. 12(a). Therefore, $t_{b}$, as defined in Fig. 12(b), is measured to 
(a)

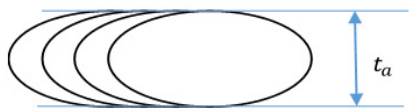

(b)

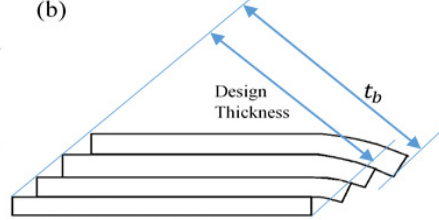

Fig. 12. Thickness of slanted struts in different directions. (a) Thickness in the direction that is not affected by $L_{c}$. (b) Thickness in the direction that is greatly affected by $L_{c}$.

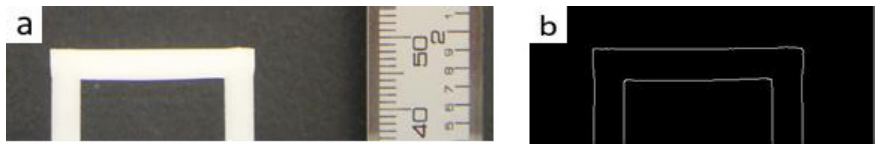

Fig. 13. Image analysis of the horizontal strut. (a) Original image. (b) Sharpened image for edge detection.

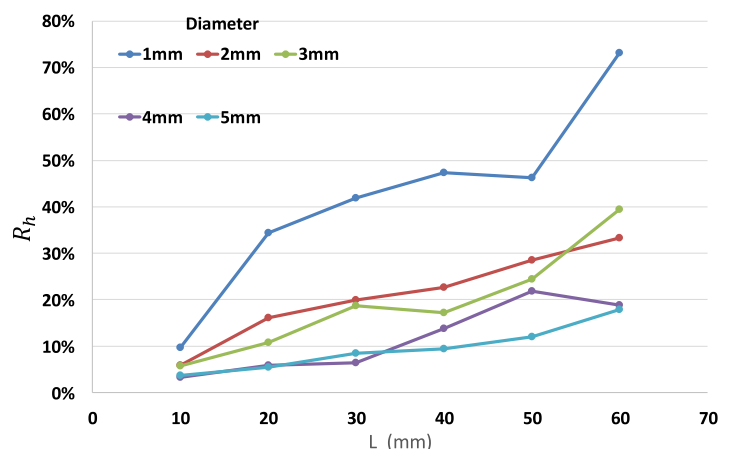

Fig. 14. Experimental result of horizontal group.

investigate the influence of an inclined angle on the deviation of the strut thickness.

b) Result of horizontal group: Because it is hard to find the largest deflection of the horizontal strut directly measured by a caliper. The image analysis method is used to find the maximum deflection point and measure it by pixels. The image of one strut is shown in Fig. 13. Fig. 13(a) shows the photograph taken by a camera. Then the image is sharpened to find the edge of the strut by MathWorks Image Processing Toolbox [41], which is shown in Fig. 13(b). A gauge block is measured by this approach to calibrate the size of the pixel. The resolution of this measurement method is $0.03 \mathrm{~mm}$. The deflection ratio $R_{h}$ is defined in (16) to illustrate the deflection of horizontal struts compared to the strut thickness

$$
R_{h}=\frac{D f}{D} \times 100 \%
$$

where $D f$ is the deflection and $D$ is the design diameter of the horizontal strut.

The result of $R_{h}$ is shown in Fig. 14. The result indicates that the $R_{h}$ is increasing with the increase of the $L$. When $D$ gets larger, the percentage of deflection will decrease. So it can be concluded that the ratio of the deflection of horizontal struts is not only related to the length of the strut, but also related to the design diameter. Therefore, when considering the manufacturability of horizontal struts, both the diameter and the length of horizontal line segments should be considered.

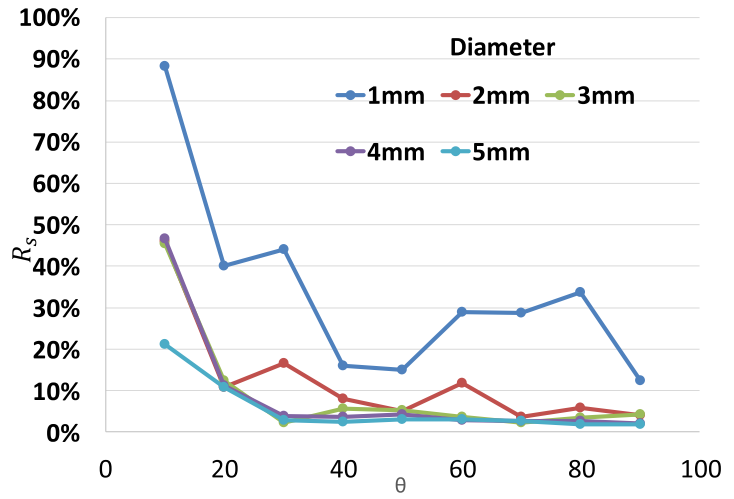

Fig. 15. Experimental result of nonhorizontal group.

c) Result of nonhorizontal group: The thickness of inclined struts $t_{b}$ is measured by a caliper with $0.01 \mathrm{~mm}$ accuracy. The ratio of the thickness deviation of slanted struts $R_{S}$ is defined in (17) to measure the discrepancy ratio compared to the designed thickness

$$
R_{s}=\left(\frac{\left|t_{b}-D\right|}{D}\right) \times 100 \%
$$

where $t_{b}$ is the thickness defined in Fig. 12(b) and $D$ is the design diameter.

The results of $R_{S}$ are shown in Fig. 15. As the plot shows, the value gets a huge increase when $\theta$ decreased to $10^{\circ}$. $R_{S}$ of 1- and 2-mm struts is higher than that of thicker struts. And 1- and 2-mm struts tend to be less stable when the $\theta$ is less than $60^{\circ}$. It can be concluded that the thickness of the discrepancy is influenced by the diameter as well as the inclined angle of the strut.

3) Artificial Neural Network: In this paper, the ANN is used to predict the manufacturability of the lattice structure and build the meta-model. ANN is a massively parallel distributed processor consists of simple processing units. Due to large scale of parallel distributed structure as well as the ability to learn and generalize, ANN has computing power to solve complex problems that are currently intractable [42].

Because the data obtained from the experiment are not sufficient to determine the manufacturability of the lattice struts with specific geometrical data. The relationship between the manufacturability and geometrical data should be more comprehensively established. Since ANN is a nonlinear model. And it is often used when the relationship between the input and output variables is not completely understood or even unknown. Therefore, it is suitable for this research to build the nonlinear relationship between the manufacturability and geometrical parameters. And this model can analyze more input parameters without much effort so that if designers want to consider the influence of process parameters as well as design parameters the ANN model is also valid to predict the result. Another advantage of ANN is that when new data are obtained in the later fabrication, it can be imported to the network to improve the performance.

Multilayer perceptrons are used for this research. Typically, the network consists of three types of layers, the input layer that collects the input data, one or more hidden layers that makes connections between the input and output layer and 

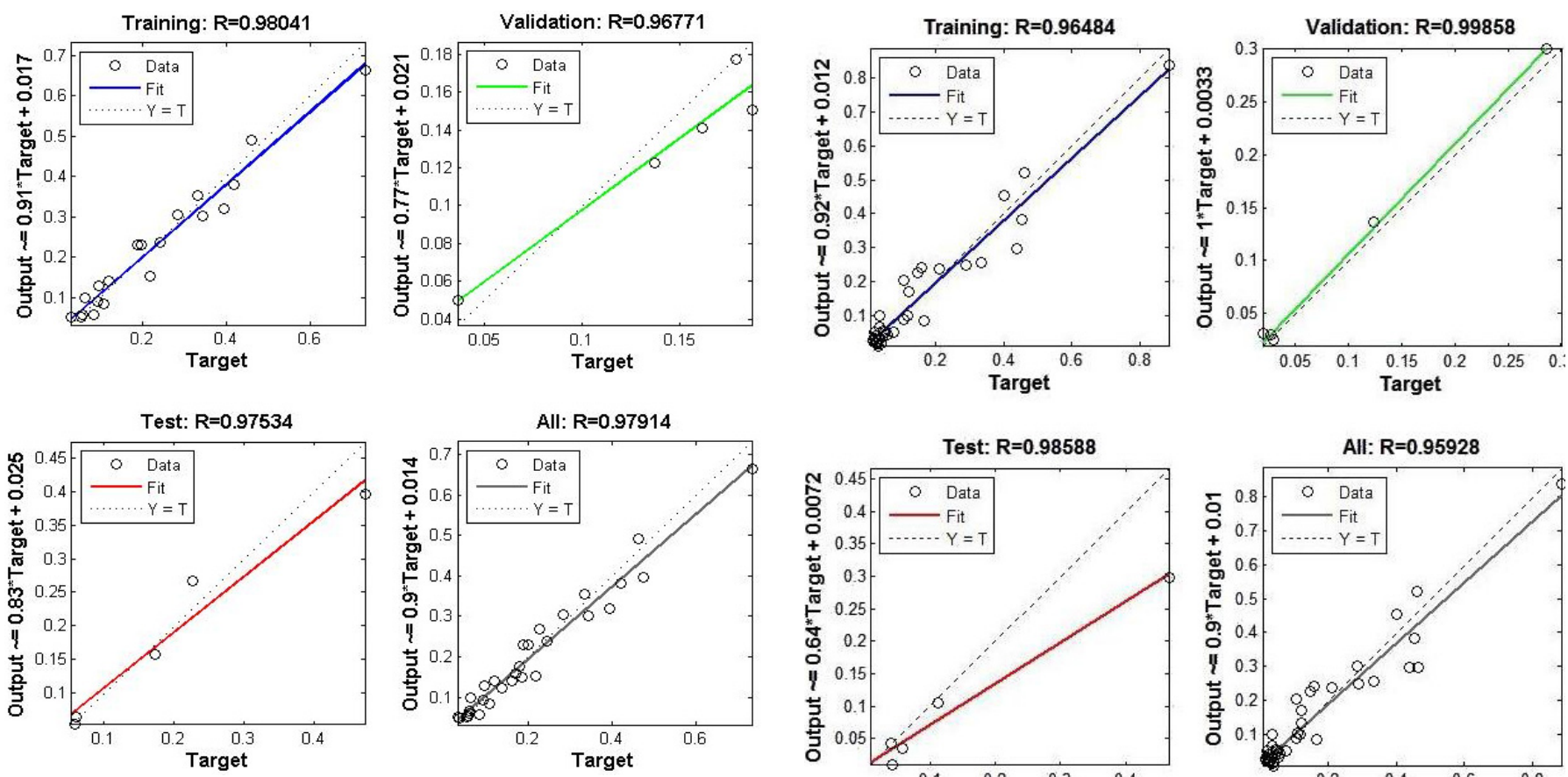

Fig. 16. Regression plot of horizontal group.
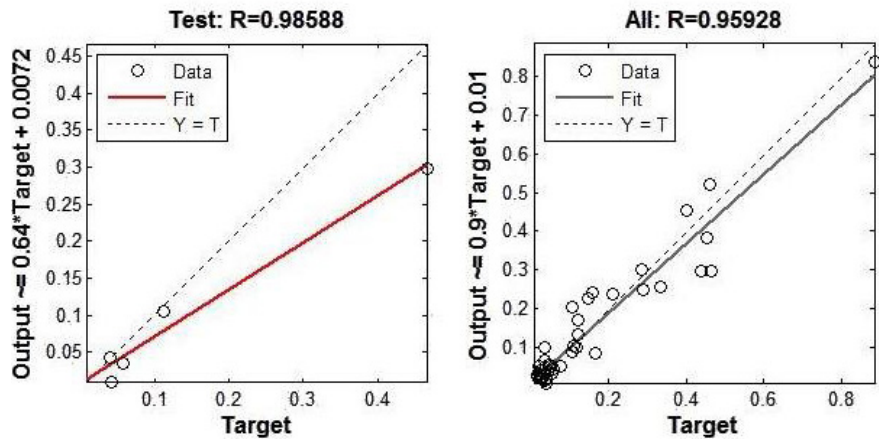

Fig. 17. Regression plot of nonhorizontal group.

analyze data, and an output layer that returns the learning results. The algorithm used for this problem is a backpropagation. It contains a forward pass which fixes the synaptic weights, and a backward pass which adjusts the synaptic weights in accordance with an error-correction rule [42]. A sigmoidal nonlinearity defined by the logistic function, as shown in (18), is used to model the nonlinear relationship

$$
y_{j}=\frac{1}{1+\exp \left(-v_{j}\right)}
$$

where $v_{j}$ is the weighted sum of all synaptic inputs plus bias of neuron $j$, and $y_{j}$ is the output of the neuron. The weights will be tuned in the training process until the error reduces to an acceptable level.

The data obtained from the experiment are trained in the MATLAB ANN tool. The input data of the horizontal group contain 30 samples. Each sample consists of 2 elements, $L$ and $D$. The output data only have one element, which is the $R_{h}$. Thirty samples are divided into the training group (22 samples), validation group (4 samples), and testing group (4 samples). The number of hidden neurons is set to be 5. Mean square error is used as the error evaluation function. And the regression $R$ values measure the correlation between outputs and targets. The regression plots across all samples for the percentage of deflection are shown in Fig. 16. It shows the relationship between the actual network outputs and the associated target values. If the linear regression fits to this output-target relationship closely alongside the line connecting the bottom-left and top-right corners of the plot, it means the network has been successfully trained to fit the data [43].

The data of the nonhorizontal group contain 45 samples. The input sample consists of two elements, the diameter and

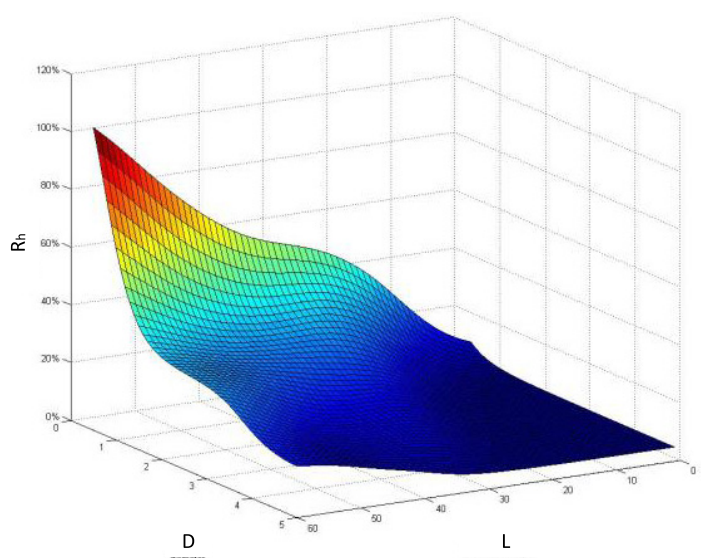

Fig. 18. Interpolation of horizontal group.

the inclined angle. The output sample only has one elements that is $R_{s}$. The training group, validation group, and testing group have 35 samples, 5 samples, and 5 samples, respectively. Other settings are the same with those of horizontal struts. The regression plot of the nonhorizontal group is shown in Fig. 17. After establishing the neural network of the input data and output data, it can be used as an interpolation approach to predict the result that was not obtained by the experiment. For the horizontal group, $D$ is interpolated from 0.5 to $5 \mathrm{~mm}$ with $0.1 \mathrm{~mm}$ as the interval, and $L$ is from 1 to $60 \mathrm{~mm}$ with $1 \mathrm{~mm}$ as the interval. The interpolation result by the neural network is shown in Fig. 18. The $z$-axis is the ANN predicted $R_{h}$. For the nonhorizontal group, $\theta$ is from $1^{\circ}$ to $90^{\circ}$ with $1^{\circ}$ as the interval. The range of the $D$ is the same as that of horizontal struts. The interpolation result is shown in Fig. 19. The $z$-axis is the ANN predicted $R_{s}$. These two interpolation plots visualize the ANNs for horizontal and nonhorizontal struts. 


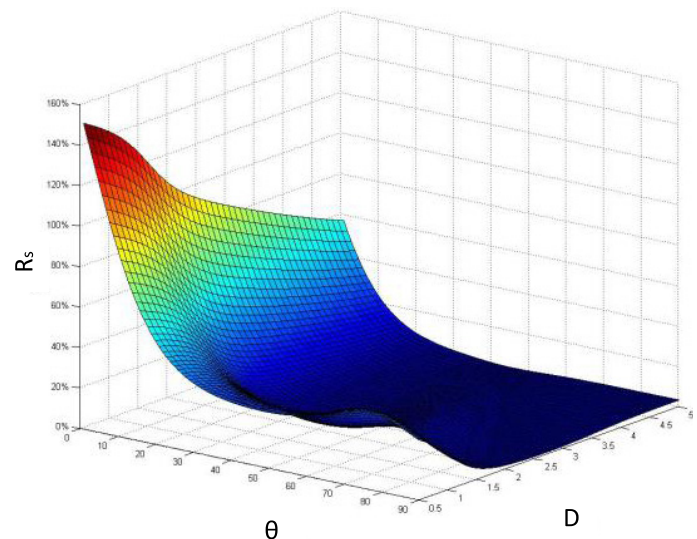

Fig. 19. Interpolation of nonhorizontal group.
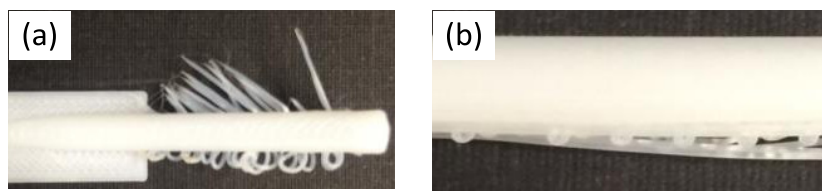

Fig. 20. (a) Inclined strut with stringing. (b) Horizontal strut with stringing.

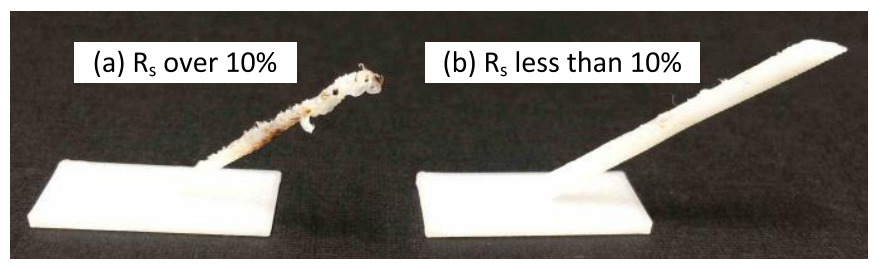

Fig. 21. Quality of inclined strut with $R_{S}$ (a) over and (b) less than $10 \%$.

If more types of geometrical data are defined, the ANNs could be more complex which may not be visualized. When using the meta-model, the first step is to choose the ANN according to the type of the strut. A user-defined quality tolerance is set for the meta-model. Particularly for this research, it is found that the horizontal struts whose deflection ratio is larger than $25 \%$ and that the vertical or slanted struts whose discrepancy ratio is larger than $10 \%$ may have the printing quality issues like stringing phenomenon as shown in Fig. 20. The filament will be detached and the printed shape is no longer the cylindrical shape as what it supposed to be. For the horizontal strut, the maximum deflection only happens at the middle region of the strut. It is found that the acceptable ratio is larger than the inclined strut. For the inclined struts, as shown in Fig. 21, it is found if the discrepancy ratio is larger than $10 \%$, the stringing phenomenon happens, and the strut gets unstable at the tip which will influence the connection between each strut. This has been verified by printing the unit cell of the strut (shown in Fig. 22). This may not significantly influence the strength of a single strut, but it may affect the quality of the following printing and the strength of the whole structure. Due to this reason, those specific values are selected as the quality tolerance in this paper. It should be noted that different designers can select different quality tolerance based on their requirements. In some special cases, more than one type of quality tolerance is needed. Then, the predefined design domain will be imported into the meta-model. Finally,

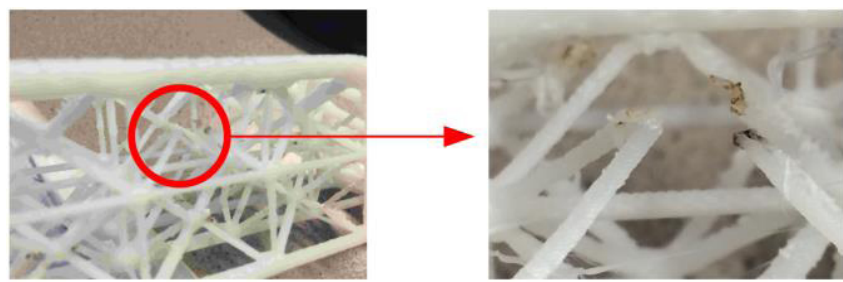

Fig. 22. Failure at the joint of the strut with $R_{S}$ equal $20 \%$.

the acceptable design domain can be determined by the quality tolerance and manufacturing constraints are obtained.

\section{F. BESO-Based Lattice Optimization}

The last step of the proposed method is to optimize the size of each strut for a given lattice frame with respect to the volume constraints from initial design stage. In this process, the manufacturing constraints from the meta-model described in Section III-E. should also be satisfied. To achieve this purpose, a BESO-based optimization algorithm is used. This algorithm is originally proposed by Querin et al [44] to update the shape and topology of structures for a better functional performance. It simulates the remodeling process of human's bone which is known as Wolff's law [45]. Recently, this algorithm has been modified and successfully applied to the design of heterogeneous lattice structures [9]. Compared to other optimization methods for lattice structures, there are three advantages of BESO method for lattice structures. First, like other heuristic optimization method, it does not require the calculation of gradients. Thus, it is easier for implementation. Second, compared to other heuristic optimization methods such as pattern search, the convergence rate can be increased by considering the stress inside the heuristic function during the design optimization process. Apart from these two advantages, the manufacturing constraints are also easier to be considered during the optimization. Due to these reasons, the BESO-based optimization method is chosen in this paper. Its mathematical representation can be expressed as

$$
\begin{aligned}
& \text { To find: } \mathbf{t}=\left(t_{1}, t_{2}, \ldots, t_{n}\right) \\
& \text { Minimize: } P I=\left(\sum_{i=1}^{i=n} \sigma_{\mathrm{VM}_{i}} V_{i}\left(t_{i}\right)\right) /(F \cdot L) \\
& \text { s.t.: } \quad K(\mathbf{t}) u-P=0 \\
& \quad \sum_{i=1}^{i=n} V_{i}\left(t_{i}\right) \leq V_{\text {const }} \\
& \quad t_{\min }^{i} \leq t_{i} \leq t_{\max }^{i}
\end{aligned}
$$

where $\mathbf{t}$ is the $n$-dimensional vector contains the size of each strut $t_{i}$ in the generated lattice frame. PI is the performance indicator of a structure to measure how well the overall structure is performing against an idealized fully stressed design [44]. $\sigma_{\mathrm{VM}_{i}}$ and $V_{i}$ represent the maximum Von-Mises stress of the $i$ th strut and its related volume, respectively. $F$ and $L$ are two parameters used to describe an idealized load case, where $F$ is a representational force and $L$ is a reference length. Their values can be predefined by designers based on the size of FV and its related loading condition. During the optimization process, the values of these two 


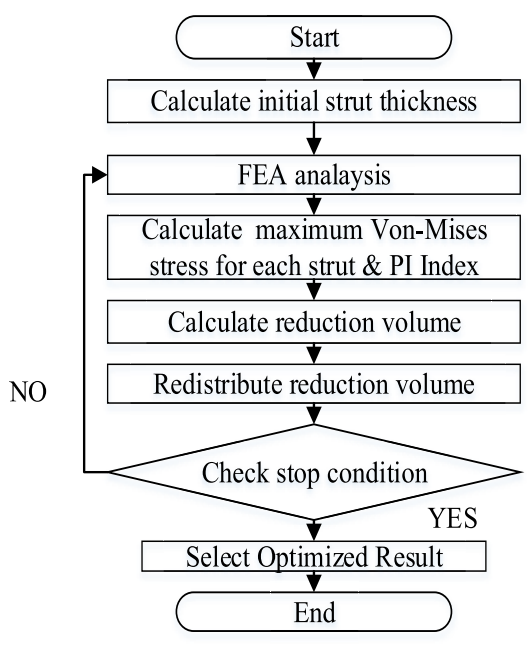

Fig. 23. General work flow of BESO-based optimization algorithm for heterogeneous lattice structures.

parameters will keep unchanged. $K$ is the global stiffness matrix of the lattice structure which can be regarded as a function of design variable $\mathbf{t}$ in this problem. $u$ is the vector of nodal displacement in lattice structures and $P$ is the nodal load. $V_{\text {const }}$ is the value of volume constraint which can be calculated from the initial design stage by $(5) . V_{i}\left(t_{i}\right)$ is a function to calculate the volume of $i$ th strut inside the lattice structure. The form of this function may vary depending on the cross-sectional shape of the strut and its length. $t_{\min }^{i}, t_{\max }^{i}$ represent the lower and upper boundary of the size of lattice struts. The minimum size of struts $t_{\min }^{i}$ may vary strut by strut, since it is mainly determined by the manufacturability of struts. Its value can be obtained from the proposed metamodel described in Section III-E. As to the upper bound of struts thickness $t_{\max }^{i}$, it is mainly controlled by the maximum allowable porosity of designed lattice structures. This value can be predefined by designers.

To solve the problem defined in the (19), the BESO-based optimization algorithm is used in this paper, and its general working flow is shown in Fig. 23. The key of this algorithm is the relocation of materials from low stress struts to high stress struts. To control this process, three parameters can be used. They are rejection ratio (RR), size remove ratio (RT), and $\mathrm{RR}$ incremental value ri. Among them, $R R$ is the ratio to control the threshold of stress for the struts whose material needs to be removed. Suppose $\sigma_{\mathrm{MAXV}}$ is the maximum Von-Mises stress of all the struts in the lattice frame, if the Von-Mises stress $\sigma_{\mathrm{VM}_{i}}$ of strut $s_{i}$ satisfy $\sigma_{\mathrm{VM}_{i}}<R R \times \sigma_{\mathrm{MAXV}}$, then a certain amount of volume should be removed from this strut until it reaches the lower bound of strut's size $t_{\min }^{i}$.

$\mathrm{RT}$ is used to control the material removal rate for lattice struts. The following equations can be used to calculate the removed volume $V_{r \text { total }}$ of each lattice strut:

$$
\begin{aligned}
t_{r_{i}} & = \begin{cases}\mathrm{RT} \times t_{i}, & \text { if } t_{i}>t_{\min }^{i} /(1-\mathrm{RT}) \\
t_{\min }^{i}, & \text { if } t_{i} \leq t_{\min }^{i} /(1-\mathrm{RT})\end{cases} \\
V_{r \text { total }} & =\sum_{i=1}^{=n}\left(A\left(t_{i}\right)-A\left(t_{i}-t_{r_{i}}\right)\right) l_{i}
\end{aligned}
$$

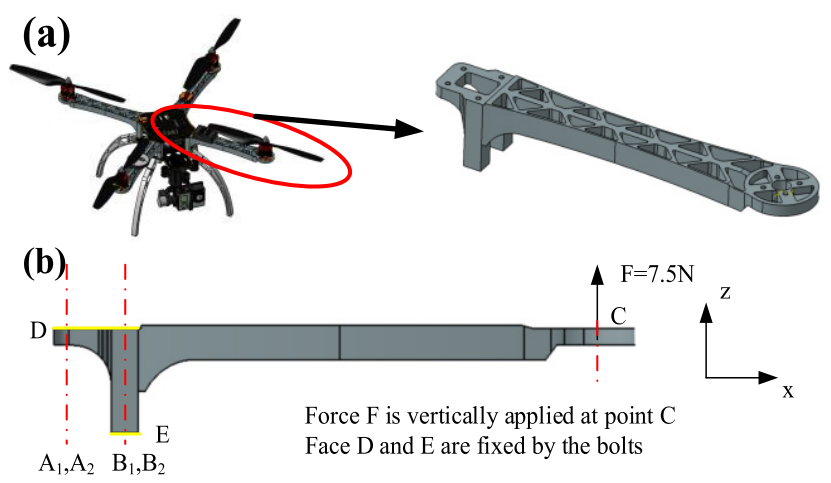

Fig. 24. Arm of quadcopter and its loading condition, (a) arm of quadcopter, original design, (b) loading condition.

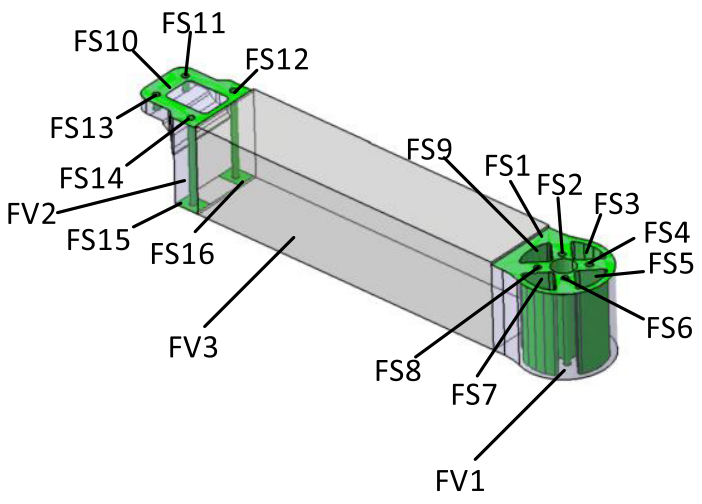

Fig. 25. Physical entity of the arm of quadcopter.

where $t_{r_{i}}$ is the target size of lattice after material removal process; $A\left(t_{i}\right)$ is the function to calculate the area of cross section with respect to the size of cross section $t_{i} . l_{i}$ is the total length of $i$ th strut.

Between two iterations, the incremental value $r i$ is used to update the current RR based on the following equation:

$$
R R^{n+1}=R R^{n}+r i
$$

where $R R^{n}$ and $R R^{n+1}$ are the $R R$ values used in the $n$th and $n+1$ th iteration, respectively.

To judge the convergence of the optimization process, the difference of PI values between two iteration steps is used. If the difference is smaller than a given value, the optimization process can be stopped. For the detailed steps of this algorithm, readers can refer the previous research paper [9].

After the optimization process, size of each lattice strut can be obtained. Based on the data and lattice frame, geometrical model of lattice structures for a given FV can be generated. To combine this model with other FVs with solid material, the final design can be obtained.

\section{Case Study and Discussion}

To further illustrate the proposed design and optimization method, a design case of the quadcopter arm is given in this section. Its original design and loading condition are shown in Fig. 24. 
TABLE II

Material Properties of ABS

\begin{tabular}{|c|c|c|c|}
\hline Elastic Modulus & Poisson Ratio & Yield Stress & Density \\
\hline $2070 \mathrm{MPa}$ & 0.35 & $38 \mathrm{Mpa}$ & $1 \mathrm{~g} / \mathrm{cm}^{3}$ \\
\hline
\end{tabular}

The major function of this part is to transfer the lifting force from the propeller to the frame of quadcopter. To achieve this function, four design requirements which are summarized based on general design and fabrication guidelines of quadcopter [46], [47] are listed in the following.

1) The maximum displacement at point $\mathrm{c}$ should be smaller than $7 \mathrm{~mm}$ under given load condition.

2) The maximum stress should be smaller than the yield stress of selected materials.

3) The total weight of part should be smaller or equal to $33 \mathrm{~g}$.

4) The main body of frame should be porous to minimize the drag force when the air passes through it vertically.

Based on the functional description and design requirements mentioned above, a physical entity with 16 FSs and 3 FVs (shown in Fig. 25) is built on the conceptual design stage. Acrylonitrile-butadiene-styrene (ABS) is selected as material for this physical entity. Its material properties used in this paper are summarized in Table II. This generated physical entity is regarded as the input of the proposed design method. Among those sixteen FSs, FS1 to FS9 are assembly surfaces for the motor, while FS10 to FS16 are assembly surfaces for the main frame of a quadcopter. To connect those FSs, three FVs are used. Among them, FV1 and FV2 are filled with solid material, while lattice structure is used in FV3 to reduce drag force when air passes through. Based on those FSs and FVs, the design space and nondesign space can be built for the topology optimization. They are shown in Fig. 26. The topology optimization formulation shown on (4) has been applied to this design and solved by an SIMP-based topology optimization solver called OptiStruct [48]. In this initial optimization process, the volume constraint has been added to control the total volume of optimized shape. The result is shown in Fig. 26. Based on this result, volume constraints of each FV are calculated by (5) and listed in Table III. Since the FV1 and FV2 are filled with the solid material, its shape can be directly obtained from the initial topology optimization process by removing the material in the low relative density region. The optimized shape of FV1 and FV2 is shown in Fig. 27. As to FV3, the volume constraint from the initial design stage is regarded as the volume constraint for lattice optimization which will be discussed in the following portion of this section.

After the initial design, the lattice frame is built to fill the FV3. To build the lattice frame, two types of parameters need to be determined. The first parameter is the lattice topology. In this case study, a cubic-center lattice cell is selected due to its stretching-dominant properties. As to cell size, general guidelines provided in Section II have been considered. Moreover, since FV3 is in a regular shape, with careful selection of cell size, it is possible to make the

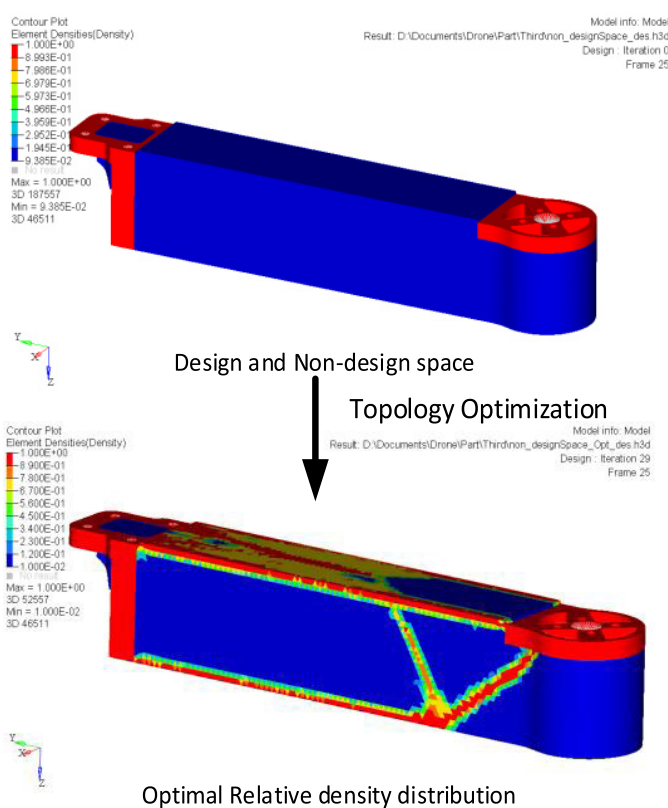

Fig. 26. Topology optimization of initial design.

TABLE III

Constraints of Volume

\begin{tabular}{|c|c|c|c|c|}
\hline & FV 1 & FV 2 & FV3 & Total \\
\hline Volume $\left(\mathrm{mm}^{3}\right)$ & 5270.60 & 7951.48 & 19777.92 & 33000 \\
\hline
\end{tabular}

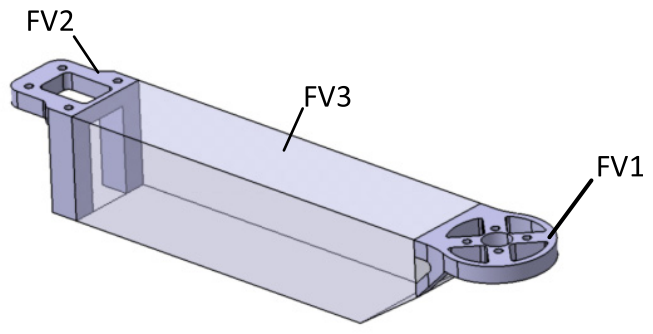

Fig. 27. Updated FVs after initial design.

TABLE IV

Dimension of THE UNit CELL

\begin{tabular}{|c|c|c|c|}
\hline Length & Width & Height & $\begin{array}{c}\text { Inclined struts' } \\
\text { angle }\end{array}$ \\
\hline $25 \mathrm{~mm}$ & $16 \mathrm{~mm}$ & $17.5 \mathrm{~mm}$ & $31^{\circ}$ \\
\hline
\end{tabular}

boundary of FV3 smooth. In other words, the FV3 can be divided by lattice cell size without remainders on the boundary. Based on these rules, the cell size is selected and summarized in Table IV, and the uniform lattice frame has been built which can full fill the FV3.

In order to provide the manufacturing constraints for the following lattice optimization process, the MEs of designed lattice frames need to be built. During the construction process of MEs, the printing orientation of lattice structures needs to be decided at first. To determine this parameter, three criteria are considered. First, to avoid the support structure 


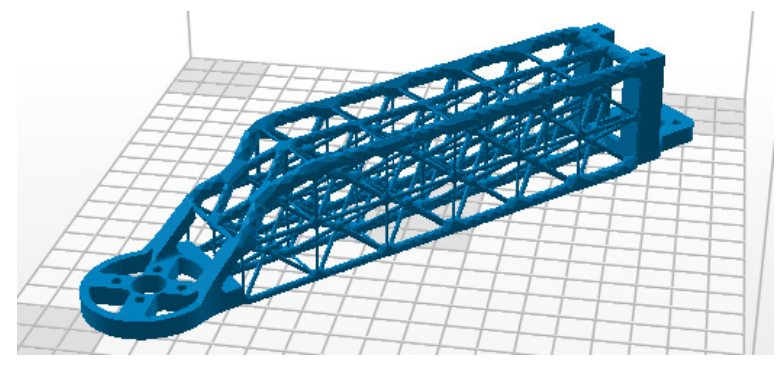

Fig. 28. Printing orientation.

TABLE V

GeOMETRICAL PARAMETERS AND CONSTRAINTS IN OPTIMIZATION ALGORITHM

\begin{tabular}{|c|c|c|c|}
\hline $\begin{array}{c}\text { Manufacturable } \\
\text { Elements }\end{array}$ & $\begin{array}{c}\text { Constant geometrical } \\
\text { data }\end{array}$ & $\begin{array}{c}\text { Lower } \\
\text { bound }\end{array}$ & $\begin{array}{c}\text { Upper } \\
\text { bound }\end{array}$ \\
\hline Horizontal strut & $\mathrm{L}=16 \mathrm{~mm}$ & $0.9 \mathrm{~mm}$ & $5 \mathrm{~mm}$ \\
\cline { 2 - 4 } & $\mathrm{L}=25 \mathrm{~mm}$ & $1.5 \mathrm{~mm}$ & $5 \mathrm{~mm}$ \\
\hline \multirow{2}{*}{ Slanted strut } & $\theta=90^{\circ}$ & $0.9 \mathrm{~mm}$ & $5 \mathrm{~mm}$ \\
\cline { 2 - 4 } & $\theta=31^{\circ}$ & $1.9 \mathrm{~mm}$ & $5 \mathrm{~mm}$ \\
\hline
\end{tabular}

in the cylindrical surface, those functional surfaces FS2-FS9 and FS11-FS14 should be perpendicular to the printing platform. Therefore, only two orientations can satisfy the first criterion. The second criterion is that for the functional volume FV1, the printing orientation should minimize the support structure. Obviously when the functional surfaces FS1 and FS10 are placed at the bottom of the platform, FV1 can be printed without supporting structures. The last criterion is that the printing layout of the model should fit the printing volume of the selected machine. Consequently, the printing orientation is determined, as shown in Fig. 28. Besides the printing orientation, the predefined design domain of struts' thickness is also determined in this stage. This predefined domain ranges from 0.6 to $5 \mathrm{~mm}$. The upper and lower bounds of this domain are determined based on the minimal resolution of the machine as well as the porosity constraints. Based on those parameters mentioned above, the MEs of designed lattice structures can be built and input into the established metal-model. the manufacturing constraints can be obtained with the given quality tolerance $\left(R_{h}\right.$ is set to be less than $25 \%$ for horizontal struts and $R_{S}$ is set to be less than $10 \%$ for slanted and vertical struts in this case study). Then the lower and upper bounds of the design domain with manufacturing constraints are found. All the geometrical parameters and constraints are summarized in Table V. The arm of the quadcopter is optimized under those manufacturability constraints and given parameters for optimization algorithm summarized in Table VI. After 52 iterations, the optimization converged (the change of the PI index is less than a given value 0.001), which is shown in Fig. 29. The CAD model of the optimized quadcopter arm shown in Fig. 30(a) is successfully fabricated [Fig. 30(b)] by the selected FDM machine. The arm of the quadcopter is also optimized without considering the manufacturability constraints of lattice structures. This part is failed during the manufacturing process.

The simulation results of different types of design configurations are summarized in Table VII. Compared to the original
TABLE VI

VAlue of Parameter Used in the Optimization Algorithm

\begin{tabular}{|c|c|c|c|}
\hline$R T$ & $r i$ & $F$ & $L$ \\
\hline 0.1 & 0.01 & $7.5 \mathrm{~N}$ & $165 \mathrm{~mm}$ \\
\hline
\end{tabular}

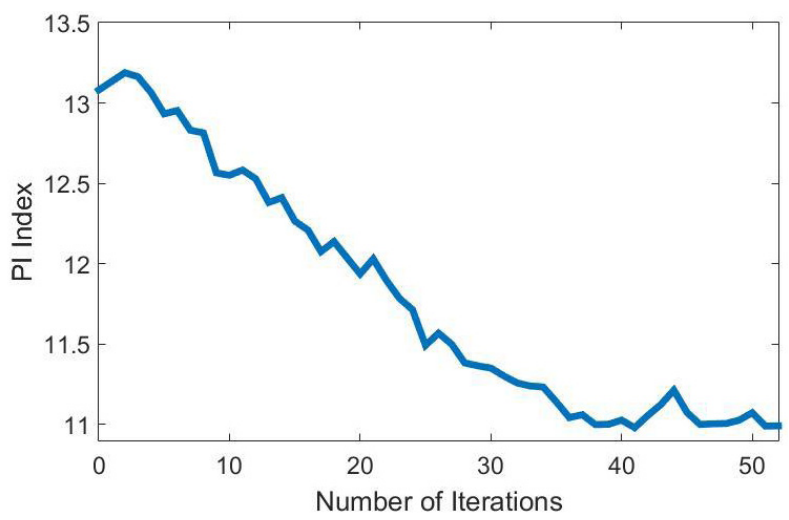

Fig. 29. PI index with respect to number of iterations.

$\mathrm{a}$
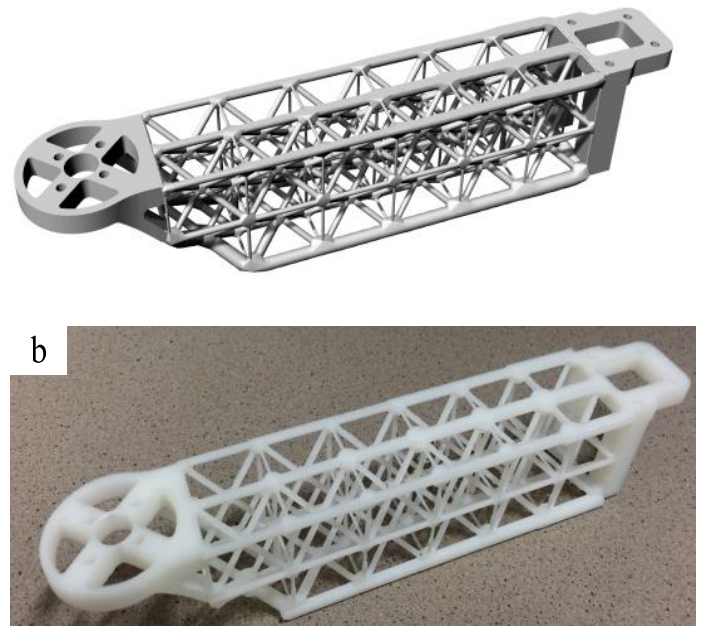

Fig. 30. Optimized quadcopter arm with manufacturing constraints. (a) CAD model. (b) FDM-fabricated part.

design and the design of homogeneous lattice structures, it is obvious that optimized heterogeneous lattice structures can significantly improve the structural stiffness without increasing its weight. To further verify this conclusion, physical tests on both homogeneous lattice and optimized heterogeneous lattice have been done. In the physical tests, a tensile test machine (ADMET MicroEP series with $45 \mathrm{~N}$ load sensor) is used to apply the given load and measure the displacement on the end of arm. A clamp is used to fix the other end of arm. The experimental setup used in this paper is shown in Fig. 31. The results of physical tests are also summarized in Table VII. It shows a small deviation (less than $0.1 \mathrm{~mm}$ ) between experimental results and physical testing results. This deviation is mainly caused by the anisotropic material properties of printed ABS material which has not been considered in the current paper. However, both simulation and experimental 
TABLE VII

COMPARISON OF SiMULATION RESULTS

\begin{tabular}{|c|c|c|c|c|c|}
\hline & $\begin{array}{c}\text { Optimization } \\
\text { with constraints }\end{array}$ & $\begin{array}{c}\text { Optimization with } \\
\text { uniform constraint }\end{array}$ & $\begin{array}{c}\text { Homogenous } \\
\text { Lattice }\end{array}$ & $\begin{array}{c}\text { Topology } \\
\text { optimization }\end{array}$ & Original design \\
\hline Volume $\left(\mathrm{mm}^{3}\right)$ & 31277 & 32534 & 32375 & 33905.15 & 33376 \\
\hline $\begin{array}{c}\text { Displacement at Point C- } \\
\text { Simulation (mm) }\end{array}$ & 0.375 & 0.319 & 0.875 & 0.354 & 4.834 \\
\hline $\begin{array}{c}\text { Displacement - Physical } \\
\text { tests (mm) }\end{array}$ & 0.454 & Not Available & 0.78 & Not Available & 4.807 \\
\hline Max Von Mises (MPa) & 1.857 & 1.690 & 4.351 & 2.42 & 15.11 \\
\hline Manufacturability & yes & no & yes & no & yes \\
\hline
\end{tabular}

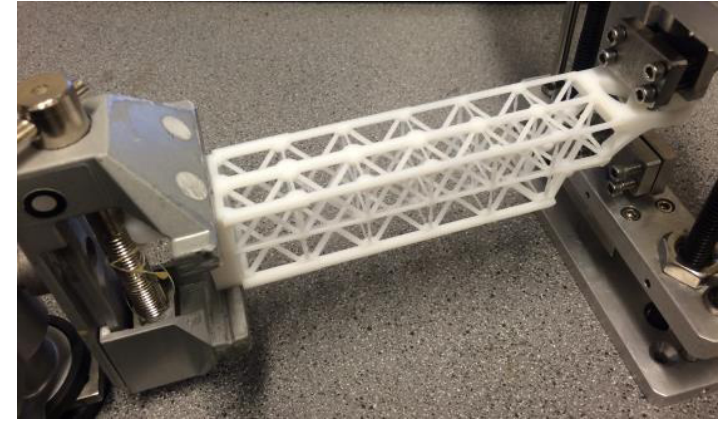

Fig. 31. Setup of physical test.

data show a clear trend that the designed lattice structure can significantly improve the structural stiffness compared to its original design (shown in Fig. 24) and homogeneous lattice. Generally, the efficiency of the proposed design method has been validated by both simulation and physical results.

To further evaluate the efficiency of the proposed lattice design and optimization method, the part [shown in Fig. 32(b)] directly obtained from traditional topology optimization routine (SIMP) has also been considered as a benchmark part in this paper. The displacement contours of optimized lattice structures and topology optimization result are compared and shown in Fig. 32. Even though the topology optimization results can achieve slightly better stiffness, the large area of the overhang region in the topology optimized part makes it difficult to be directly fabricated without support structure. Moreover, as it is shown in Table VII, optimized lattice structures may achieve a smaller maximum Von-Mises stress compared to topology optimization result.

Another interesting fact which can be observed from Table VII is that the optimized lattice with uniform constraint can achieve a better stiffness than that of nonuniform constraints obtained from the proposed method as well as the result of topology optimization. In this paper, the uniform constraint refers smallest cylinders that can be printed by the selected machine. Its value is $0.6 \mathrm{~mm}$ for the selected machine. This constraint is uniformly applied to all the struts during the optimization process. Compared to the optimized lattice with nonuniform constraints, the uniform constraints can provide a larger design freedom for designers. Thus, the structure can be further optimized to achieve better performance. However, this structure cannot be fabricated with the selected machine, since the dimension of optimized struts violates the constraints

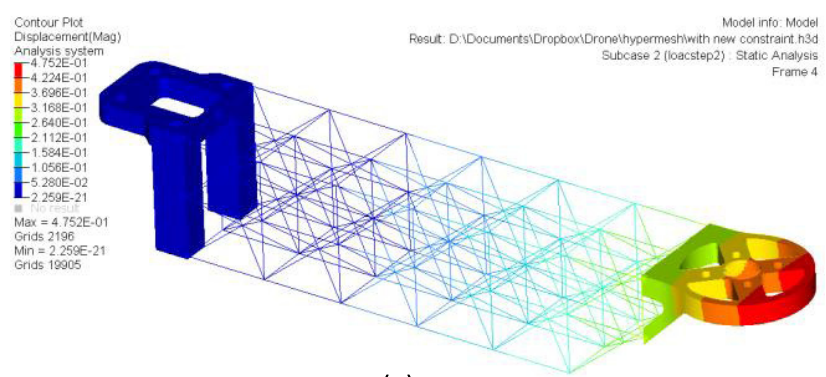

(a)

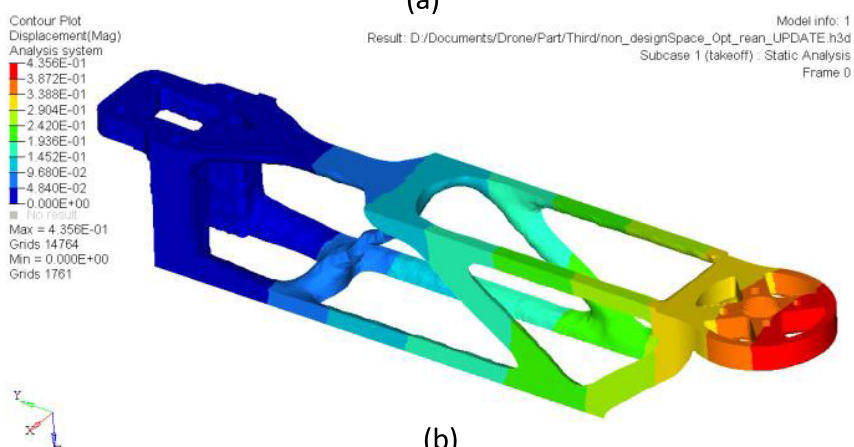

(b)

Fig. 32. Comparison between optimized lattice structures and topology optimization result. (a) Displacement contour of optimized lattice structures. (b) Displacement contour of topology optimization result.

summarized in Table V. Based on this fact, it can be inferred that if the process related parameters, such as printing strategy and process parameters, can be optimized to alleviate the existing manufacturing constraints, the performance of parts can be further improved by the proposed design method.

\section{CONClusion}

In this paper, a design method of lattice structures under the manufacturability constraints of AM process has been proposed. The meta-model for the selected AM process is obtained from experiments and ANN. A BESO-based optimization process is used to find the optimum struts' thickness distribution.

Several conclusions can be drawn from this paper are as follows.

1) The lattice structures generated by the proposed design method can improve the stiffness of the model. Von-Mises stress and displacement can be reduced without increasing the volume. 
2) The heterogeneous lattice structure optimized by the proposed method has a better performance compared to the homogenous lattice structure.

3) The meta-model obtained from the experiment and ANN has ensured the manufacturability of the lattice structure by certain AM process.

4) If the design domain can be enlarged, which in this paper means that if the lower bound of the struts' thickness can be smaller, the performance can be further improved.

Future research will be focused on the following prospects. First, relations between struts' geometrical dimensions and mechanical properties need to be further investigated. This relation can provide a feedback to lattice simulation and optimization model with more accurate material properties. Second, other two aspects of MEs including the material data and the process data, can be further investigated in the future research to build a more comprehensive meta-model for lattice structures. Finally, the process parameter can be optimized to obtain a larger feasible area for design and optimization.

\section{REFERENCES}

[1] Merriam-Webster. (2016). Lattice. [Online]. Available: http://www. merriam-webster.com/dictionary/lattice

[2] V. S. Deshpande, N. A. Fleck, and M. F. Ashby, "Effective properties of the octet-truss lattice material," J. Mech. Phys. Solids, vol. 49, no. 8, pp. 1747-1769, 2001.

[3] J. Lehman and R. Lakes, "Stiff lattices with zero thermal expansion and enhanced stiffness via rib cross section optimization," Int. J. Mech. Mater. Design, vol. 9, no. 3, pp. 213-225, 2013.

[4] L. Yang, D. Cormier, H. West, O. Harrysson, and K. Knowlson, "Nonstochastic Ti-6Al-4V foam structures with negative Poisson's ratio," Mater. Sci. Eng. A, vol. 558, pp. 579-585, Dec. 2012.

[5] Q. Zhang, Y. Han, C. Chen, and T. Lu, "Ultralight X-type lattice sandwich structure (I): Concept, fabrication and experimental characterization," Sci. China, E, Technol. Sci., vol. 52, no. 8, pp. 2147-2154, 2009.

[6] P. Heinl, L. Müller, C. Körner, R. F. Singer, and F. A. Müller, "Cellular Ti-6Al-4V structures with interconnected macro porosity for bone implants fabricated by selective electron beam melting," Acta Biomaterialia, vol. 4, no. 5, pp. 1536-1544, Sep. 2008.

[7] S. Bose, S. Vahabzadeh, and A. Bandyopadhyay, "Bone tissue engineering using 3D printing," Mater. Today, vol. 16, no. 12, pp. 496-504, Dec. 2013.

[8] C. Emmelmann, P. Scheinemann, M. Munsch, and V. Seyda, "Laser additive manufacturing of modified implant surfaces with osseointegrative characteristics," Phys. Procedia, vol. 12, Part A, pp. 375-384, 2011.

[9] Y. Tang, A. Kurtz, and Y. F. Zhao, "Bidirectional evolutionary structural optimization (BESO) based design method for lattice structure to be fabricated by additive manufacturing," Comput.-Aided Design, vol. 69, pp. 91-101, Dec. 2015.

[10] D. W. Rosen, "Computer-aided design for additive manufacturing of cellular structures," Comput.-Aided Design Appl., vol. 4, no. 5, pp. 585-594, 2007.

[11] Z. Ozdemir et al., "Energy absorption in lattice structures in dynamics: Experiments," Int. J. Impact Eng., vol. 89, pp. 49-61, Mar. 2016.

[12] J. Lehman and R. S. Lakes, "Stiff, strong, zero thermal expansion lattices via material hierarchy," Compos. Struct., vol. 107, pp. 654-663, Jan. 2014.

[13] H. Brooks and K. Brigden, "Design of conformal cooling layers with self-supporting lattices for additively manufactured tooling," Additive Manuf., vol. 11, pp. 16-22, Jul. 2016.

[14] H. N. G. Wadley, "Multifunctional periodic cellular metals," Philos. Trans. Roy. Soc. London A, Math. Phys. Sci., vol. 364, no. 1838, pp. 31-68, 2006.

[15] H. Wang, Y. Chen, and D. W. Rosen, "A hybrid geometric modeling method for large scale conformal cellular structures," in Proc. ASME Comput. Inf. Eng. Conf., Long Beach, CA, USA, 2005, pp. 421-427.

[16] J. Panetta, Q. Zhou, L. Malomo, N. Pietroni, P. Cignoni, and D. Zorin, "Elastic textures for additive fabrication," ACM Trans. Graph., vol. 34, no. 4, p. 135, 2015.
[17] C. Yan, L. Hao, A. Hussein, and D. Raymont, "Evaluations of cellular lattice structures manufactured using selective laser melting," Int. J. Mach. Tools Manuf., vol. 62, pp. 32-38, Nov. 2012.

[18] M. Santorinaios, W. Brooks, C. J. Sutcliffe, and R. A. Mines, "Crush behaviour of open cellular lattice structures manufactured using selective laser melting," WIT Trans. Built Environ., vol. 85, pp. 481-489, 2006.

[19] A. Hussein, L. Hao, C. Yan, R. Everson, and P. Young, "Advanced lattice support structures for metal additive manufacturing," J. Mater. Process. Technol., vol. 213, no. 7, pp. 1019-1026, Jul. 2013.

[20] D. Wang, Y. Yang, R. Liu, D. Xiao, and J. Sun, "Study on the designing rules and processability of porous structure based on selective laser melting (SLM)," J. Mater. Process. Technol., vol. 213, no. 10, pp. 1734-1742, Oct. 2013.

[21] Z. Ming, F. Raphael, S. Yaw-Kang, T. Harold, and B. Jeffrey, "Progress in topology optimization with manufacturing constraints," in Proc. 9th AIAA/ISSMO Symp. Multidisciplinary Anal. Optim. Amer. Inst. Aeronautics Astron., 2002, p. 5614.

[22] J. Yan, G.-D. Cheng, and L. Liu, "A uniform optimum material based model for concurrent optimization of thermoelastic structures and materials," Int. J. Simul. Multidisciplinary Design Optim., vol. 2, no. 4, pp. 259-266, 2008.

[23] J. Deng, J. Yan, and G. Cheng, "Multi-objective concurrent topology optimization of thermoelastic structures composed of homogeneous porous material," Struct. Multidisciplinary Optim., vol. 47, no. 4, pp. 583-597, Apr. 2013.

[24] X. Yan, X. Huang, Y. Zha, and Y. M. Xie, "Concurrent topology optimization of structures and their composite microstructures," Comput. Struct., vol. 133, pp. 103-110, Mar. 2014.

[25] Y. Tang and Y. F. Zhao, "Design method for lattice-skin structure fabricated by additive manufacturing," presented at the ASME Int. Mech. Eng. Congr. Expo., Montreal, QC, Canada, 2014, p. V02BT02A030.

[26] V. G. Sundararajan, "Topology optimization for additive manufacturing of customized meso-structures using homogenization and parametric smoothing functions," M.S. thesis, Dept. Mech. Eng., Univ. Texas Austin, Austin, TX, USA, 2011.

[27] D. Brackett, I. Ashcroft, and R. Hague, "A dithering based method to generate variable volume lattice cells for additive manufacturing," in Proc. 22nd Annu. Int. Solid Freeform Fabrication Symp., Austin, TX, USA, Aug. 2011, pp. 8-10.

[28] R. Rezaie, M. Badrossamay, A. Ghaie, and H. Moosavi, "Topology optimization for fused deposition modeling process," Procedia CIRP, vol. 6, pp. 521-526, 2013.

[29] D. Brackett, I. Ashcroft, and R. Hague, "Topology optimization for additive manufacturing," in Proc. 22nd Anпи. Int. Solid Freeform Fabrication Symp., 2011, pp. 348-362.

[30] J. Wu, C. C. L. Wang, X. Zhang, and R. Westermann, "Self-supporting rhombic infill structures for additive manufacturing," Comput.-Aided Design, vol. 80, pp. 32-42, Nov. 2016.

[31] J. Nguyen, S.-I. Park, and D. Rosen, "Heuristic optimization method for cellular structure design of light weight components," Int. J. Precis. Eng. Manuf., vol. 14, no. 6, pp. 1071-1078, Jun. 2013.

[32] Y. Tang, Y. Sheng, and Y. F. Zhao, "Design method for conformal latticeskin structure fabricated by AM technologies," presented at the ASME Int. Design Eng. Tech. Conf. Comput. Inf. Eng. Conf., Charlotte, NC, USA, 2016, p. V01AT02A037.

[33] Y. Tang, S. Yang, and Y. F. Zhao, "Sustainable design for additive manufacturing through functionality integration and part consolidation," in Handbook of Sustainability in Additive Manufacturing, vol. 1, S. S. Muthu and M. M. Savalani, Eds. Singapore: Springer, 2016, pp. 101-144.

[34] Y. Tang, J.-Y. Hascoet, and Y. F. Zhao, "Integration of topological and functional optimization in design for additive manufacturing," in Proc. ASME 12th Biennial Conf. Eng. Syst. Design Anal., 2014, p. V001T06A006.

[35] Y. Tang, K. Mak, and Y. F. Zhao, "A framework to reduce product environmental impact through design optimization for additive manufacturing," J. Cleaner Prod., vol. 137, pp. 1560-1572, Nov. 2016.

[36] M. P. Bendsøe, A. Díaz, and N. Kikuchi, "Topology and generalized layout optimization of elastic structures," in Topology Design of Structures. Dordrecht, The Netherlands: Springer, 1993, pp. 159-205.

[37] H. V. Wang, "A unit cell approach for lightweight structure and compliant mechanism," Ph.D. dissertation, Dept. Mech. Eng., Georgia Inst. Technol., Atlanta, GA, USA, 2005.

[38] V. S. Deshpande, M. F. Ashby, and N. A. Fleck, "Foam topology: Bending versus stretching dominated architectures," Acta Mater, vol. 49, no. 6, pp. 1035-1040, Apr. 2001. 
[39] M. F. Ashby, Y. J. M. Bréchet, D. Cebon, and L. Salvo, "Selection strategies for materials and processes," Mater. Design, vol. 25, no. 1, pp. 51-67, Feb. 2004.

[40] Z-ABS Material Data Sheet, Olsztyn, Poland, Zortrax Inc, 2015.

[41] (2016). MathWorks Image Processing Toolbox Documentation. [Online]. Available: http://www.mathworks.com/help/images/index.html

[42] S. Haykin, Neural Networks: A Comprehensive Foundation. Englewood Cliffs, NJ, USA: Prentice-Hall, 1998

[43] (2016). MathWork MATLAB R2016b Documentation. [Online]. Available: http://www.mathworks.com/help/nnet/examples/house-priceestimation.html

[44] O. M. Querin, G. P. Steven, and Y. M. Xie, "Evolutionary structural optimisation (ESO) using a bidirectional algorithm," Eng. Comput., vol. 15 , no. 8, pp. 1031-1048, 1998.

[45] H. M. Frost, "Wolff's Law and bone's structural adaptations to mechanical usage: An overview for clinicians," Angle Orthodontist, vol. 64, no. 3, pp. 175-188, 1994.

[46] A. V. Javir, K. Pawar, S. Dhudum, N. Patale, and S. Patil, "Design, analysis and fabrication of quadcopter," J. Int. Assoc. Adv. Technol. Sci., vol. 16, no. 11, 2015.

[47] I. Gaponov and A. Razinkova, "Quadcopter design and implementation as a multidisciplinary engineering course," in Proc. IEEE Int. Conf. Teaching, Assessment Learn. Eng. (TALE), Aug. 2012, pp. H2B-16-H2B-19.

[48] A. E. Inc, Optistruct User's Guide V12.0, Altair Engineering Inc, Troy, MI, USA, 2013

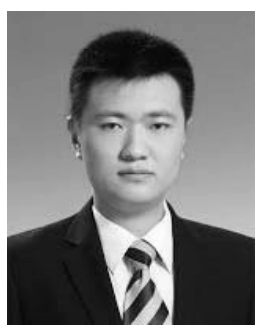

Yunlong Tang was born in Chongqing, China. He received the B.Eng. degree in mechanical engineering from the Harbin Institute of Technology, Harbin, China, in 2010, and the M.Eng. degree from Beihang University, Beijing, China, in 2013. He is currently pursuing the Ph.D. degree with the Department of Mechanical Engineering, McGill University, Montreal, QC, Canada.

His research interests mainly focus on computeraided design and manufacturing, additive manufacturing and its related design methods and tools. His current research topic is the design methodology for additive manufacturing process.

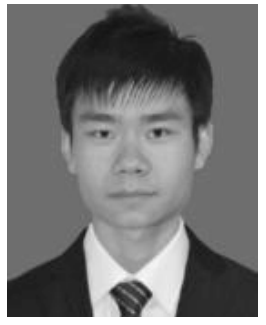

Guoying Dong was born in Luoyang, Henan, China, in 1993. He received the B.S. degree in aerospace engineering from Northwestern Polytechnical University, Xi' an, China, in 2015. He is currently pursuing the Ph.D. degree in mechanical engineering with McGill University, Montreal, QC, Canada.

His research interests include the finite-element analysis of lattice structures, manufacturability of lattice structures, and the characteristics of additive manufacturing on the mechanical performance.

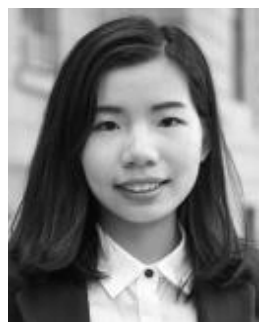

Qinxue Zhou was born in Wuhan, China, in, 1997. She has been pursuing the B.Eng. (Hons.) degree in mechanical engineering with McGill University, Montreal, QC, Canada, since 2014.

Her research interests is mostly focused on additive manufacturing and design optimization.

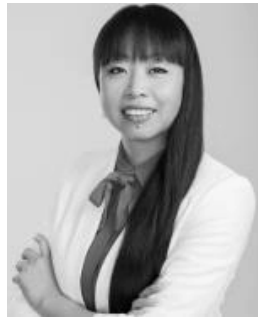

Yaoyao Fiona Zhao received the B.E. from the Beijing Institute of Technology, Beijing, China, in 2003, and the M.E. (First Class Hons.) and Ph.D. degrees from the University of Auckland, Auckland, New Zealand, in 2006 and 2010, respectively.

She was a Researcher at the National Institute of Standards and Technology, Gaithersburg, MD, USA, and a Post-Doctoral Research Fellow at Ecole Centrale de Nantes, France, from 2010 to 2012. She is currently an Assistant Professor with the Department of Mechanical Engineering, McGill University, Montreal, QC, Canada, where she is also the Head of the Additive Design and Manufacturing Laboratory. Her research expertise lies in design and manufacturing systems integration, quality control and optimization. More specifically, her research focuses on manufacturing informatics, dimensional metrology, additive manufacturing design and process modeling, as well as manufacturing sustainability analysis. 